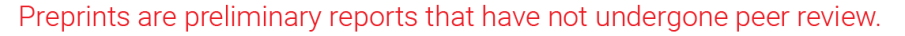 They should not be considered conclusive, used to inform clinical practice, or referenced by the media as validated information. \\ Issue of Lithium-indium Anode in High Energy and Power All-Solid-State Lithium Batteries
}

\section{Shuting Luo}

Tsinghua University

\section{Zhenyu Wang}

Guilin Electrical Equipment Scientific Research Institute Co. Ltd.

\section{Xuelei Li}

Tianjin University of Technology

\section{Xinyu Liu}

Guilin Electrical Equipment Scientific Research Institute Co. Ltd.

\section{Haidong Wang}

Tsinghua University

\section{Weigang Ma}

Tsinghua University https://orcid.org/0000-0003-0178-3994

\section{Lianqi Zhang}

Tianjin University of Technology

\section{Lingyun zhu}

Guilin Electrical Equipment Scientific Research Institute Co. Ltd.

\section{Xing Zhang ( $\nabla$ x-zhang@tsinghua.edu.cn )}

Tsinghua University

\section{Article}

Keywords: all-solid-state lithium battery, sulfide solid electrolyte, Li-In dendrites, Li dendrites

Posted Date: April 22nd, 2021

DOI: https://doi.org/10.21203/rs.3.rs-402674/v1

License: (c) (1) This work is licensed under a Creative Commons Attribution 4.0 International License. Read Full License

Version of Record: A version of this preprint was published at Nature Communications on November 29th, 2021. See the published version at https://doi.org/10.1038/s41467-021-27311-7. 


\section{Issue of Lithium-indium Anode in High Energy and Power All-Solid-State Lithium Batteries}

Abstract: All-solid-state lithium batteries (ASSLBs) using sulfide solid electrolytes (SSEs) offer an attractive option for energy storage applications. Lithium anode is the ultimate goal for ASSLBs, but lithium-indium (Li-In) alloy anode is more widely utilized in lab testing owing to the quite stable interface and elimination for the risk of short circuit. However, vigorous growth of Li-In dendrites in SSE is discovered in the present work when a full cell $\left(\mathrm{LiNbO}_{3}\right.$ coated $\left.\mathrm{LiNi}_{0.6} \mathrm{Co}_{0.2} \mathrm{Mn}_{0.2} \mathrm{O}_{2} / / \mathrm{Li}_{6} \mathrm{PS}_{5} \mathrm{Cl} / / \mathrm{Li}-\mathrm{In}\right)$ is cycled in high loading and high rate. Our study demonstrates that Li-In anode is unstable towards SSEs at high current, which induces Li-In dendrite growth enclosing electrolyte particles and eventually results in cell death after a long cycling. The morphology and growth mechanism of Li-In dendrites are revealed by scanning transmission electron microscopy-electron energy loss spectroscopy (STEM-EELS) analysis and density function theory (DFT) calculations. Moreover, the differences between Li and Li-In dendrites are systematically compared.

Keywords: all-solid-state lithium battery; sulfide solid electrolyte; Li-In dendrites; Li dendrites

\section{Introduction}

The thermal instability of conventional lithium-ion batteries (LIBs) which originated from the intrinsic characteristics of liquid electrolytes causes safety issues and has 
1 become a serious impediment to the automotive application. All-solid-state lithium

2 batteries (ASSLBs) using nonflammable solid electrolytes may not only overcome the

3 safety concerns in LIBs but also achieve high energy density ${ }^{1,2,3,4,5}$. Lithium metal is

4 recognized as the most attractive choice for anode material to achieve high energy

5 density due to the low electrochemical potential (-3.04 V vs. the standard hydrogen

6 electrode) and high theoretical specific capacity (3860 $\left.\mathrm{mAh} \mathrm{g}^{-1}\right)$. However, pure Li

7 metal is unsatisfying in sulfide-based ASSLBs because of the severe interfacial side

8 reactions $6,7,8$ and the growth of Li dendrites $9,10,11$. Lithium alloys provide an attractive

9 alternative to construct a stable SSEs-electrode interface that enables the long-term

cycling for ASSLBs ${ }^{12}$. Lithium alloys can be easily prepared by solid-state diffusion method at ambient temperature. A number of alloys including $\mathrm{Li}-\mathrm{Al}{ }^{13}, \mathrm{Li}-\mathrm{In}^{14}{ }^{15}$, Li$\mathrm{Si}^{16}, \mathrm{Li}-\mathrm{Au}{ }^{17,18,19}, \mathrm{Li}-\mathrm{Sn}{ }^{20}$ have been reported as interlayers or solid solution for sulfide-based ASSLBs. Generally, alloy layer or bulk has higher lithium diffusivity than pure lithium, which is favorable for lithium transport towards interface and thus a uniform lithium plating can be achieved ${ }^{21,22}$. In addition, the insertion of lithium into other metals can reduce the lithium chemical potential and suppress the electrochemical decomposition of SSEs ${ }^{23,24}$. mechanical ductility and constant redox potential (about $0.6 \mathrm{~V}$ vs. $\mathrm{Li}^{+} / \mathrm{Li}$ ) over a wide stoichiometry range ${ }^{15}$. Li-In alloy is usually considered as a thermodynamically and kinetically stable material towards SSEs and is widely used in the laboratory for testing the performance of electrolytes or cathodes. Li-In alloys exhibit excellent long-cycling 

$2\left(<0.5 \mathrm{~mA} \mathrm{~cm}^{-2},<0.5 \mathrm{C}\right)^{25,26,27}$, it is unclear whether or not Li-In alloy anode is still 3 stable towards SSEs at high current, which is critical for the high power applications of

4

stability towards SSEs in ASSLBs. However, most batteries were cycled at low current ASSLBs. There are rare investigations to clarify the issue.

In the present work, we cycled a full cell at high loading $\left(4 \mathrm{mAh} \mathrm{cm}^{-2}\right)$ and high rate (1C) to investigate the interface stability between sulfide electrolyte and $\mathrm{Li}$-In anode. Unexpectedly, the cell has a short circuit after 897 cycles, which is similar as using Li metal anode. Combined with scanning electron microscope (SEM) and scanning transmission electron microscope (STEM) observations, we discovered the growth of Li-In dendrites in LPSCl solid electrolyte, which leads to a rapid capacity fading and subsequent battery failure. The underlying mechanism for Li-In dendrite growth is revealed by electron energy loss spectroscopy (EELS) analysis and ab initio molecular dynamics (AIMD) simulations. The differences between $\mathrm{Li}$ and $\mathrm{Li}-\mathrm{In}$ dendrites in morphology and growth mechanism are also compared.

\section{Results and discussion}

\subsection{Cell failure for ASSLBs using Li-In alloy}

The purity of synthesized LPSCl was determined by XRD patterns (Figure S1 in supporting information). All the diffraction peaks are well indexed to the pure phase $\mathrm{Li}_{7} \mathrm{PS}_{6}$. The electrolyte LPSCl has a high ion conductivity of $2.95 \times 10^{-3} \mathrm{~S} \mathrm{~cm}^{-1}$ at room temperature (RT) as measured by electrochemical impedance spectroscopy (Figure S2). The NCM622 particles were uniformly coated by LNO with around $10 \mathrm{~nm}$ thickness as 
1 shown in Figure S3. The SEM image of the cross section of prepared Li-In alloy is

2 shown in Figure S4 with alloy phase circled by blue dotted lines. Due to the good creep

3 property of lithium, Li metal diffuses into the In metal and the formed alloy phase is

4 uniformly distributed in indium matrix.

5 Figure 1a shows the long-term cycling of the assembled cell

6 LNO@NCM622//LPSCl//Li-In at 1C at RT with a high loading of $4 \mathrm{mAh} \mathrm{cm}^{-2}$. The

7 cell maintains a stable cycling capacity and near $100 \%$ columbic efficiency during the

8 charge-discharge cycle up to 890 cycles (Figure 1a). However, the capacity started to

9 decline after 891 cycles, and finally the discharge capacity decreased to $\sim 0$ at the 897 th

cycle. Figure $1 \mathrm{~b}$ displays the related charge-discharge voltage profile from the $891^{\text {th }}$ to

11 the $897^{\text {th }}$ cycle of the cell, in which the charge specific capacity increased gradually

12 while the corresponding discharge specific capacity decreased. At the $897^{\text {th }}$ cycle, the

13 cell was continuously charged with everlasting capacity increase accompanying a lower

14 voltage increasing rate as illustrated in Figure 1c, which indicates the appearance of

15 internal short circuit and cell death. These results are similar to those of using lithium anode, which indicates that Li-In alloy anode is not forever stable for sulfide electrolyte, especially after a longer charge-discharge cycle with a high current density. 


\section{a}

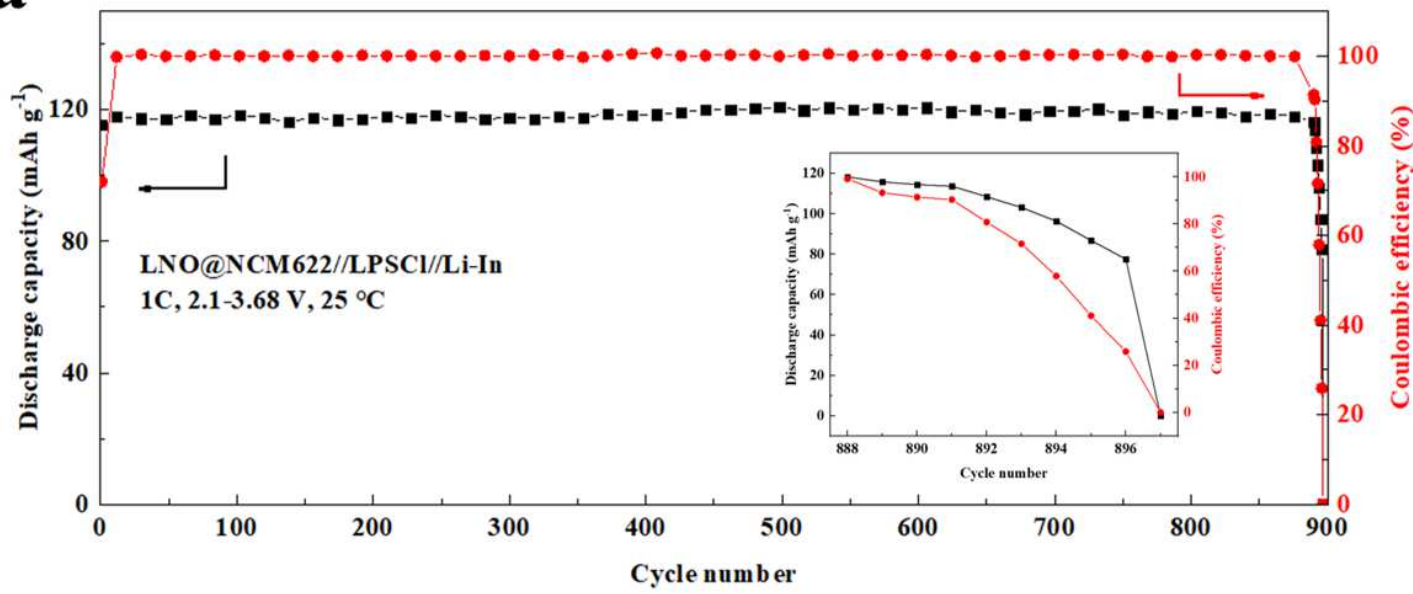

b
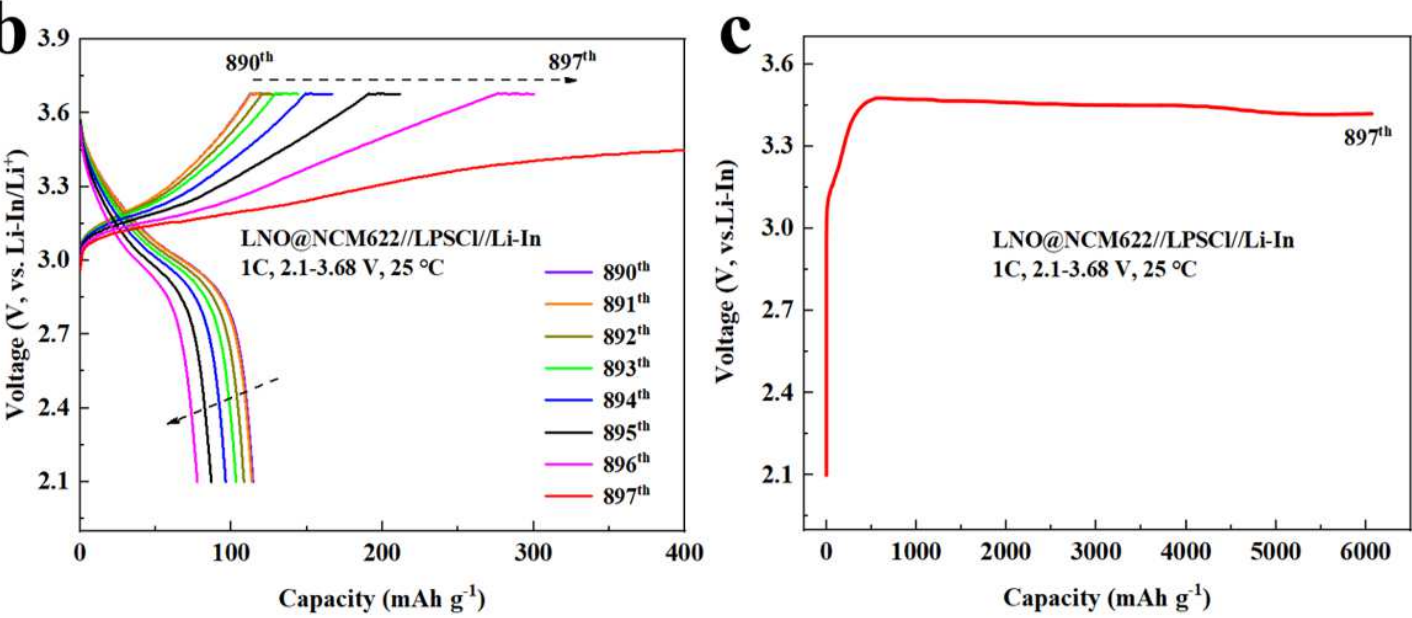

Figure 1. (a) Long-term cycling of the assembled cell LNO@NCM622//LPSCl//Li-In at 1C at RT.

(b) Galvanostatic charge-discharge profiles from the $890^{\text {th }}$ to the $897^{\text {th }}$ cycle. (c) Galvanostatic charge profile for the $897^{\text {th }}$ cycle.

\subsection{Dendrite growth of Li-In alloy in sulfide solid electrolyte}

In order to find out the reason for the cell failure using Li-In alloy anode, we conducted SEM observations for the cells with different cycling numbers. Figure 2a shows the cross-sectional SEM image for the fresh cell before cycling. LNO@NCM622 cathode, LPSCl electrolyte and Li-In anode can be clearly distinguished from the SEM images at backscattered electron (BSE) mode. The magnified image for the boxed region indicates an intimate contact between electrolyte and anode. 


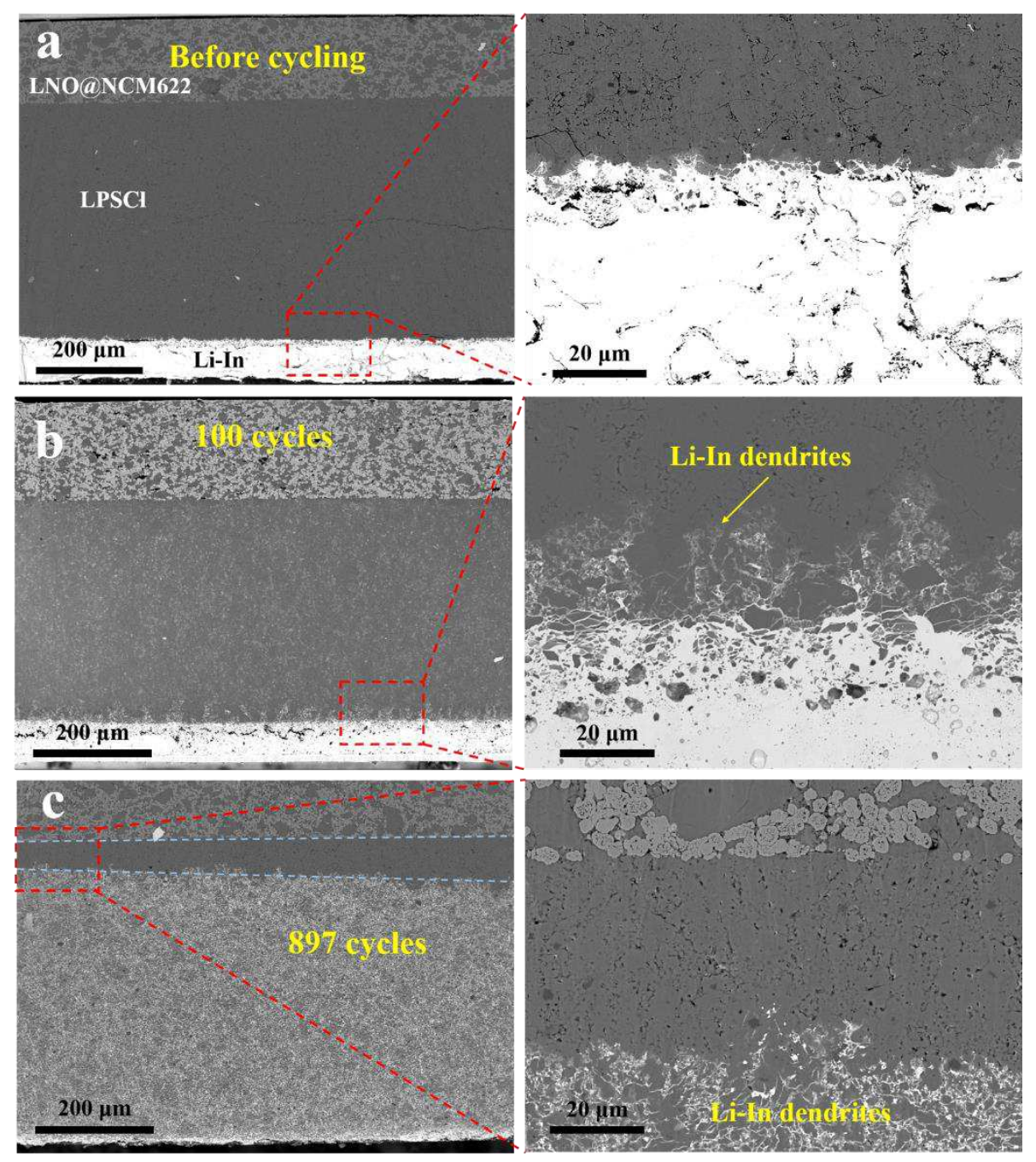

2 Figure 2. Cross-sectional SEM images for the cell LNO@NCM622//LPSCl//Li-In (a) before

3 cycling, (b) after 100 cycles and (c) after 897 cycles.

$4 \quad$ For the cell cycled 100 times shown in Figure 2b, different from the fresh state, Li-

5 In alloy grows into the electrolyte for around $20 \mu \mathrm{m}$ and exhibits flame shape at the

6 anode-electrolyte interface. We called the Li-In anode that grows into the electrolyte as

7 Li-In dendrites. For the dead cell after 897 cycles, the Li-In alloy exhibits a striking

8 growth towards electrolyte interior with around $500 \mu \mathrm{m}$, nearly having a contact with

9 cathode. Li-In alloy almost entirely enters into the electrolyte without significant anode

10 layer observed. Due to the growth inhomogeneity of Li-In dendrites at different

11 positions and limited area of observation, Li-In alloy has definitely penetrated through 
1 the electrolyte at some locations and results in short circuit and cell death. In the

2 meantime, there is no significant structure or composition change for cathode and

3 cathode-electrolyte interface as demonstrated in Figure S5.Therefore, the cell failure of

$4 \quad$ ASSLB using Li-In anode at high current is induced by the growth of Li-In dendrites.

5 To deeply analyze the morphology of Li-In dendrites in LPSCl electrolyte, SEM and

6 EDX observations were conducted at various positions for the cell cycled 897 times.

7 The SEM image at the middle of Li-In dendrites (red boxed area in Figure 3a) are

8 displayed in Figure 3b. It is clearly observed that the Li-In dendrites grow densely and

9 laterally in stripes, which is further demonstrated by the EDX mapping of indium 10 element (Figure 3f). Elements of P, S, Cl (Figure 3c-3e) are uniformly distributed in the 11 LPSCl electrolyte. Although the Li-In alloy grows wildly in the electrolyte, there are 12 no obvious cracks and voids observed. The high compactness of the electrolyte 13 indicates that the Li-In dendrites have lower growth stress than Li dendrites without 14 severe structural damage for electrolyte itself. 

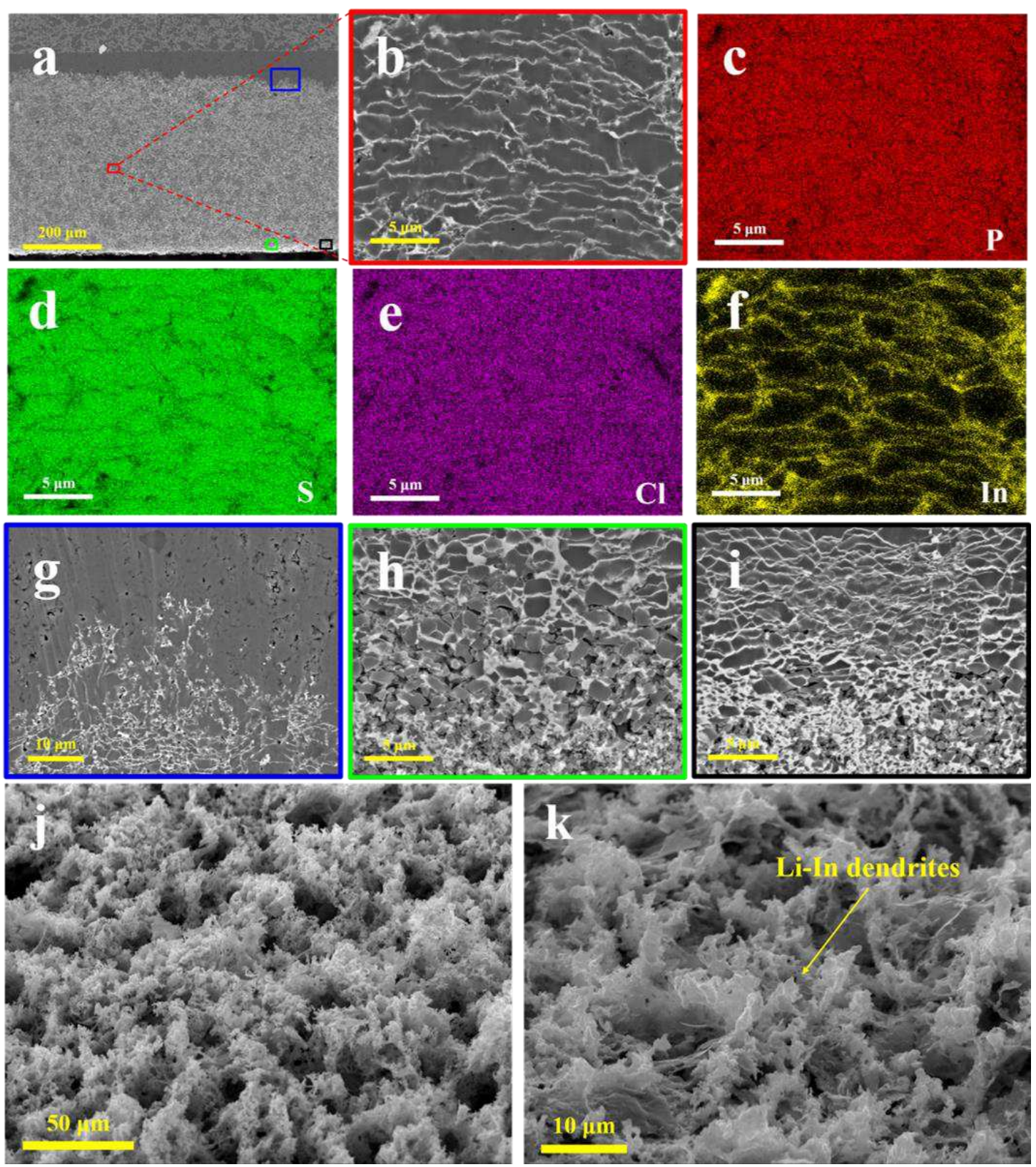

2 Figure 3. (a) Cross-sectional SEM images of the cell LNO@NCM622//LPSCl//Li-In after 897

3 cycles. (b) SEM image and EDX mapping of (c) P, (d) S, (e) Cl and (f) In at the middle of Li-In

4 dendrites. SEM images at (g) the top of Li-In dendrites, (h) the In-rich layer and (i) the interface

5 between electrolyte and In-rich layer. SEM images of Li-In dendrites from an oblique view after the

6 cell was soaked and washed with the deionized water at (j) low magnification and (k) high

7 magnification.

8 Figure $3 \mathrm{~g}$ shows the morphology at the top of Li-In dendrites (blue boxed area in 
1 Figure 3a). Different from the streak pattern at the middle of Li-In dendrites, it displays

2 a flame shape, which is similar as the morphology shown in Figure $2 \mathrm{~d}$ after 100 cycles.

3 From the perspective of time and space, it can be inferred that flame shape is the initial

4 morphology of Li-In dendrites. At the very bottom of the Li-In dendrites, there remains

5 an In-rich layer with around $10 \mu \mathrm{m}$ thickness (white region). We performed SEM

6 characterizations for the In-rich layer (Figure 3h, green boxed area) and the In-rich

7 layer-electrolyte interface (Figure 3i, black boxed area), respectively. As seen from

8 Figure 3c, the electrolyte is broken into smaller particles with less than $4 \mu \mathrm{m}$ diameter.

9 The particle diameter becomes smaller with getting closer to the bottom. Li-In dendrites exhibit a form of network enclosing the broken electrolyte particles. Two kinds of morphologies of Li-In dendrites appear at the In-rich layer-electrolyte interface, as shown in Figure 3i. The upper part exhibits streak pattern, which is consistent with the morphology at the middle of Li-In dendrites. The lower part shows network formation, which is in accord with In-rich layer. Therefore, it is in a transition state. influence of electrolyte, a part of the dismantled specimen of the LNO@NCM622//LPSCl//Li-In cell was soaked and washed in deionized water for 2 hours and then dried for 24 hours, by which the LPSCl electrolyte and cathode materials can be removed via chemical reaction. Figure $3 \mathrm{j}$ and $3 \mathrm{k}$ shows the SEM images of LiIn alloy anode from the oblique view at low and high magnifications, respectively. Obviously, Li-In dendrites grow densely and uniformly over a wide region, like a honeycomb that wraps the electrolyte particles in them. 
Based on the above results, it can be concluded that Li-In alloy is unstable towards

2 SSEs when cycled at a high current even though it exhibits excellent stability at low

3 current. The formed Li-In dendrites can penetrate the solid electrolyte after a long cycle

4 and eventually results in short circuit and cell failure. From the top to the bottom of Li-

5 In dendrites, the morphology changes from flame shape, to the stripe and then to the

6 network, which has a similar evolution in time with the increase of cycling number. It

7 should be noted that striped dendrites occupy the majority and this morphology is

8 favorable for reducing the growth rate of $\mathrm{Li}$-In dendrites. More importantly, these three

9 types of Li-In dendrites have one thing in common, that is they have no significant

10 destructive effect on electrolyte structure. No apparent cracks are observed even the

11 electrolyte particles were divided into smaller particles in the In-rich layer. The

12 electrolyte layer maintains a high density overall. Li-In dendrites mainly grow along

13 the grain boundaries and have a great wettability with electrolyte particles.

\subsection{Growth mechanism for Li-In dendrites}

Indium metal is commonly considered as a stable material towards sulfide electrolyte, so it is widely used for ASSLBs testing. Besides, metal In do not participate in electrochemical cycling in lithium ion batteries. How does it violently grow in SSEs? In order to find out the reason, STEM characterization were performed to reveal the growth mechanism for Li-In dendrites. Figure 4a and 4b show the STEM images of LiIn dendrites at low and high magnifications, respectively. It is clearly illustrated that there exists a $15 \mathrm{~nm}$-thick interphase layer at the In-LPSCl interface. As observed from the STEM image and EDX mapping shown in Figure 4c and 4d, there exists an obvious 
1 change of element contents in the interphase layer. Elements of P, S, Cl in the electrolyte

2 and element of In exhibit an opposite variation trend at the interphase layer. Due to the

3 high content of element S in LPSCl, the interphase layer is mainly composed of indium

4 and sulfur elements. Therefore, some indium-sulfur compounds may be generated at

5 the interphase layer. In addition, the interphase layer has an intimate contact with

6 electrolyte as observed from Figure $4 \mathrm{~b}$, which enables the high compactness of

7 electrolyte during the growth of Li-In dendrites.
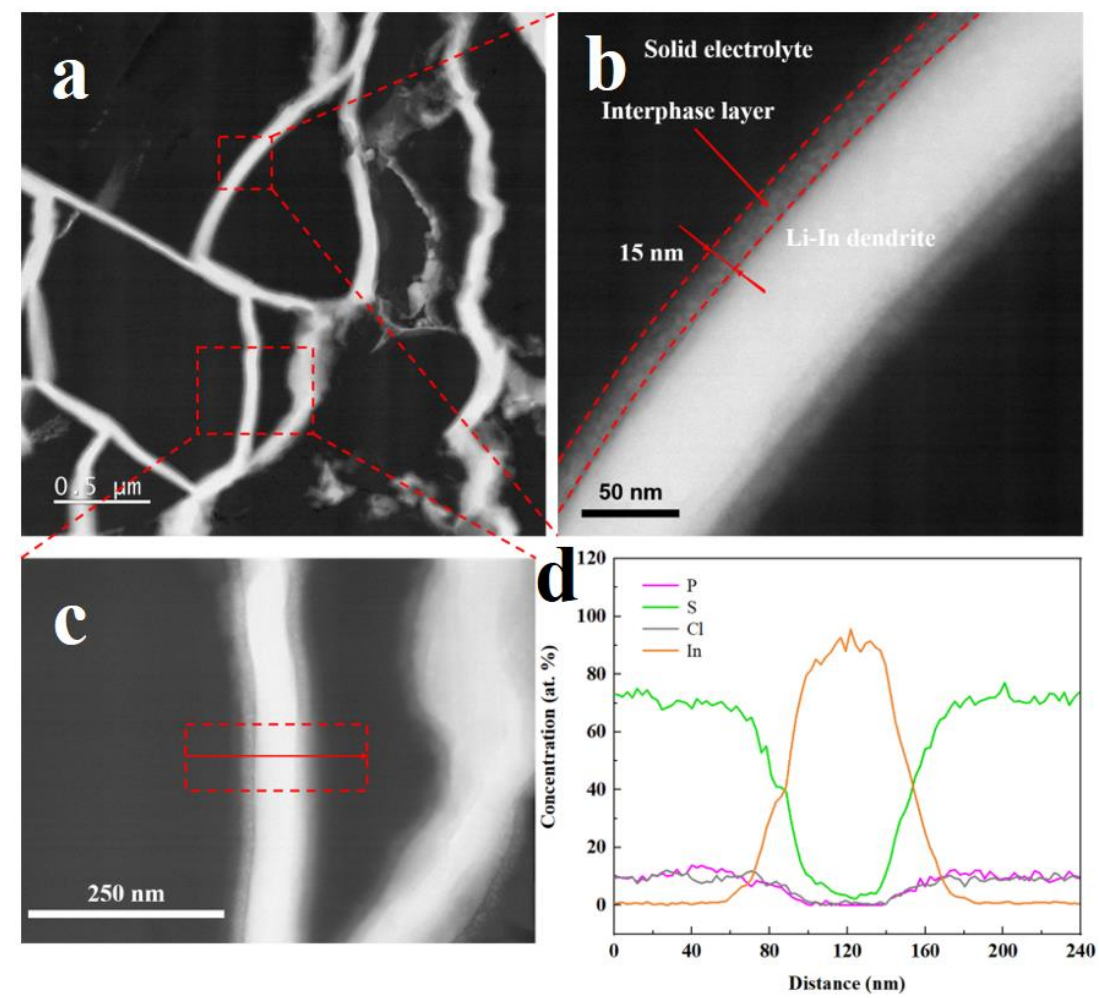

the direction of the red arrow.

2 STEM-EELS analysis was conducted to further determine the component of interphase layer. Figure 5a shows the STEM images for Li-In dendrites and corresponding EELS mapping of In and S for the magnified boxed area. The element 
1 distributions of the interphase layer are more clearly observed from the EELS mapping.

2 The variation trends of In and S in the direction perpendicular to the anode-electrolyte

3 interface agrees well with EDX mapping shown in Figure 4d. The electron energy loss

4 spectra for Li-In dendrite, LPSCl electrolyte and their interphase layer are shown in

$5 \quad$ Figure 5b. A new phase that different from metal In and LPSCl electrolyte is generated

6 at the interphase layer, which provides a direct evidence for interfacial reaction.
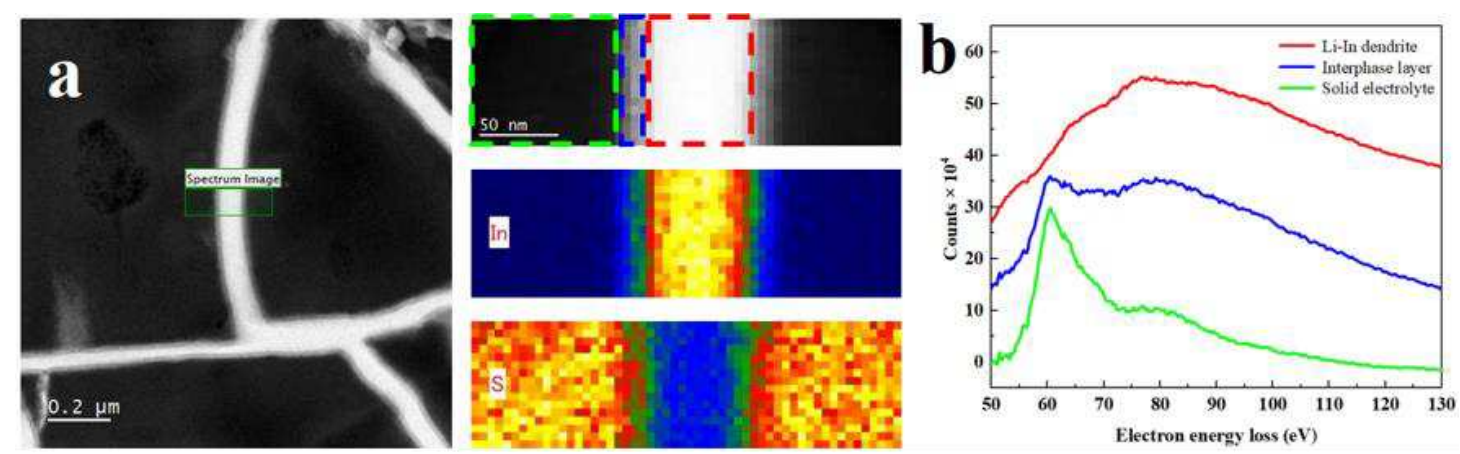

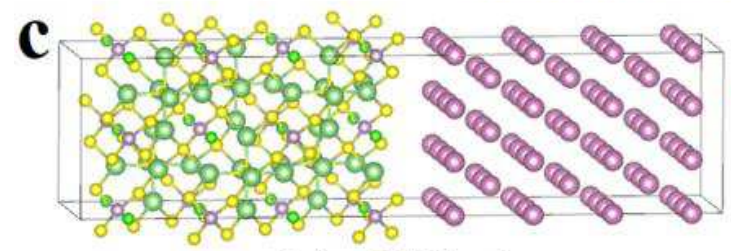

Before MD (0 ps)
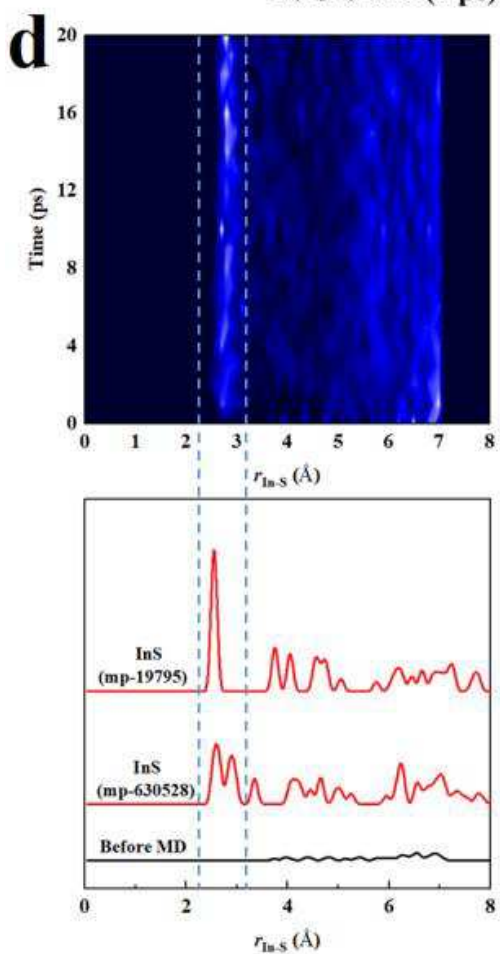
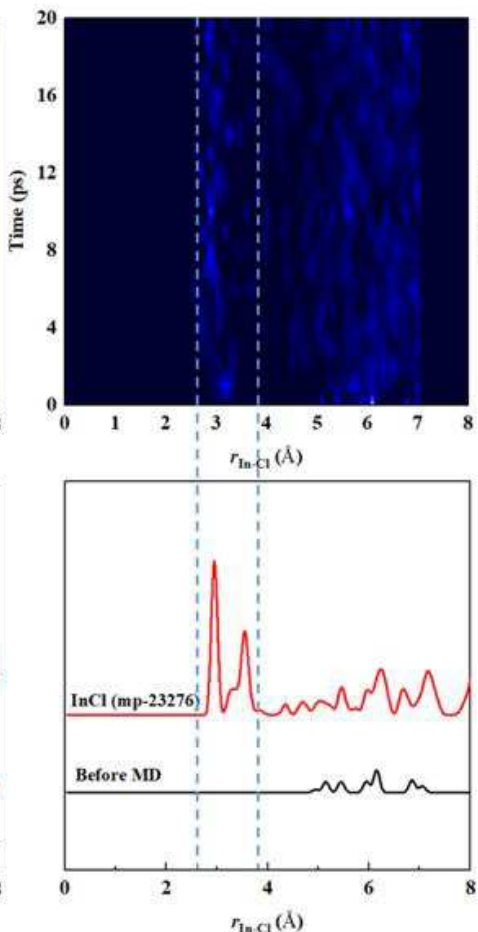

After MD (20 ps)
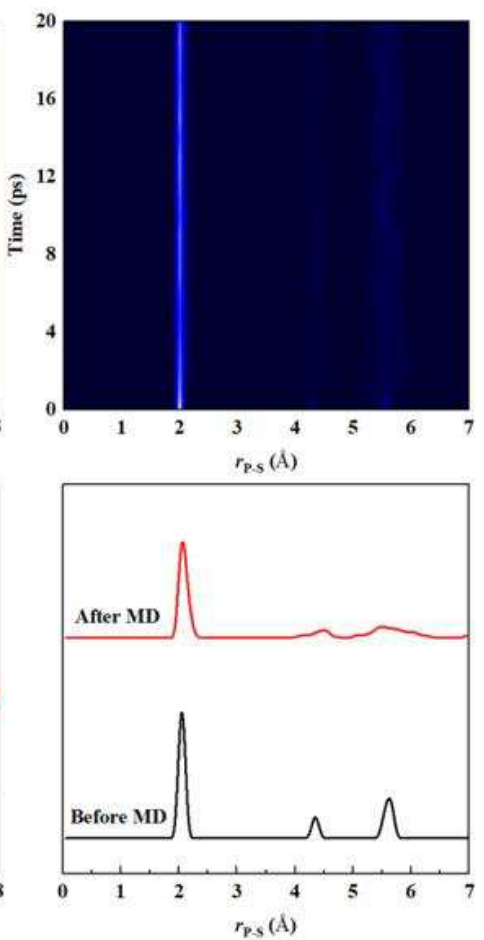

Figure 5. (a) STEM images of Li-In dendrites and corresponding EELS mapping of In and S for 
the magnified boxed area. (b) Electron energy loss spectra for Li-In dendrite, LPSCl electrolyte and their interphase layer. (c) LPSCl-In interface models before MD (0 ps) and after MD (20 ps). (d) Evolutions of RDFs of In-S, In-Cl and P-S for LPSCl-In interface during simulation and RDFs of In-S, In-Cl and P-S for reference materials ( $\mathrm{InS}, \mathrm{InCl})$ with the initial structure (before MD) provided as a reference.

Then, first-principles calculations were further performed to investigate the interface reaction between metal In and LPSCl electrolyte. The dynamic changes of LPSCl-In interface were simulated using AIMD at $300 \mathrm{~K}$ and the structural variation was tracked by radial distribution function (RDF). Figure $5 \mathrm{c}$ shows all the formed bonds after AIMD (20 ps) as well as the interface model before AIMD (0 ps). It can be clearly found that quite a number of In-S bonds and a small amount of $\mathrm{In}-\mathrm{Cl}$ bonds were formed after MD at the LPSCl-In interface. The upper part in Figure 5d shows the evolutions of In-S, In$\mathrm{Cl}$ and P-S pairs during the first 20 ps. The lower part plots the In-S RDF of InS, In-Cl $\mathrm{RDF}$ of $\mathrm{InCl}$, and $\mathrm{RDF}$ of the initial structure (before $\mathrm{MD}$ ) provided as a reference. The structures of known crystalline compounds ( $\mathrm{InS}$ and $\mathrm{LnCl}$ ) were obtained from Materials Project (MP) database. At the very beginning of the simulation, no In-S and In- $\mathrm{Cl}$ bonds are found matching those in $\mathrm{InS}$ and $\mathrm{InCl}$. The initial formation of In-S and In- $\mathrm{Cl}$ bonds is at $\sim 1 \mathrm{ps}$. During the simulation, the intensity of In-S bonds $(\sim 2.8 \AA)$ gradually increases and then keep stable, indicating the formation of stable reaction product InS. However, the intensity of In-Cl bonds (2.9 $\AA$ ) has been fluctuating during $20 \mathrm{ps}$, which means $\mathrm{InCl}$ is an unstable intermediate product and easily decomposes. 
much less than that of InS, which can be reflected from the number of In-S bonds and In-Cl bonds in Figure 5c. Therefore, InS should be the main reaction product.

EELS results and AIMD simulations demonstrates that chemical side reaction occurs at the In-LPSCl interface and the generation of InS is thermodynamically favorable. However, whether the growth of Li-In dendrites can continue depends not only on thermodynamic favorability, but also on kinetic feasibility. The growth rate of $\mathrm{Li}-\mathrm{In}$ dendrites is closely related to the charging-discharging current. When the cell is cycled at a high current, a large amounts of lithium ions enters into the indium matrix during charging, which will induce the volume expansion of dendrite tip. Obviously, grain boundary is the preferential expansion channel due to the least resistance. In addition, the new produced InS at the interface improves the wettability behavior between Li-In and LPSCl particles, which results in an intimate contacting interface and honeycomb dendrite structure. Therefore, the formed Li-In dendrites fills the grain boundaries like liquid and tightly enclose the electrolyte particles, which enables the structural stability of the electrolyte.

\subsection{Comparison between Li-In dendrites and Li dendrites}

Lithium dendrites have been widely investigated in recent decades, however, the formation of Li-In dendrites is firstly reported in this work. In order to find out the difference between $\mathrm{Li}$ and $\mathrm{Li}$-In dendrites, we performed corresponding electrochemical measurements and SEM observations for LNO@NCM622//LPSCl//Li cell. The cycling performance and the galvanostatic charge-discharge profiles are shown in Figure S6. Compared with the cell using Li-In anode, it has a much shorter 
1 cycling life (17 cycles) and a much lower charge/discharge current $\left(0.3 \mathrm{~mA} \mathrm{~cm}{ }^{-2}\right)$. A

2 similar short circuit occurs in the last few cycles caused by the growth of Li dendrites.

3 The charge capacity gradually increases, while discharge capacity and coulombic

4 efficiency simultaneously decreases and finally to zero.

5 Figure 6 a presents the cross-sectional SEM images for the fresh

6 LNO@NCM622//LPSCl//Li cell without cycling. The cracks are caused by the cell

7 disassembly, which is common in non-binder cell. The magnified image of the boxed

8 area includes a part of the LPSCl electrolyte adjacent to Li anode. The interphase layer

9 with "grey" at the interface is evidently different from Li anode and LPSCl electrolyte

10 observed from the high-contrast BSE image, indicating a severe interfacial side reaction

11 between Li and LPSCl. After 17 cycles as shown in Figure 6b, the growth of Li

12 dendrites is much sharper than that of $\mathrm{Li}$-In dendrites and exhibits a different growth

13 morphology. Li dendrites not only grow along the grain boundaries, but also destroy

14 the electrolyte structure and induces much cracks, which results in a rapid short circuit 15 within several cycles. 

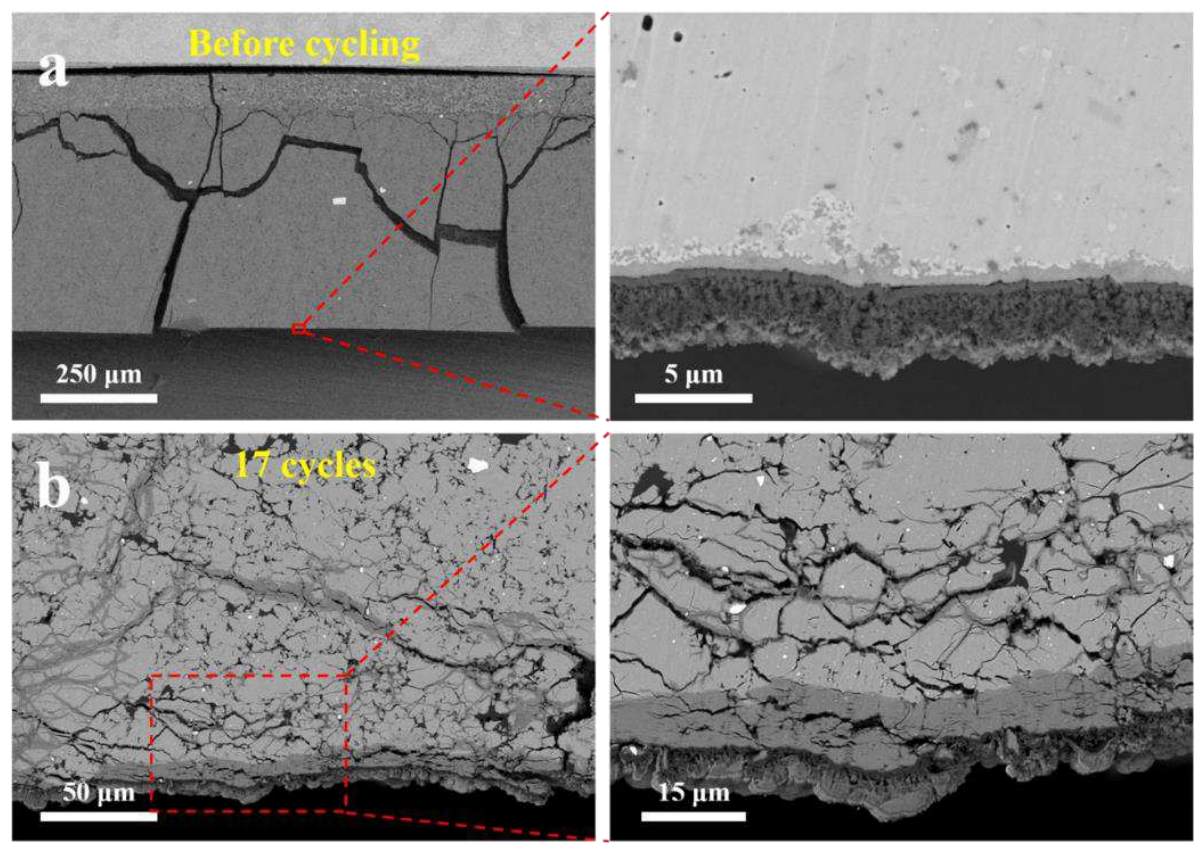

2 Figure 6. Cross-sectional SEM images of the cell LNO@NCM622//LPSCl//Li (a) before cycling and (b) after 17 cycles.

4 Combined with the Li-In dendrites shown above, we can find that there exist

5 significant differences between Li-In and Li dendrites. Firstly, they have different growth morphologies. Lithium dendrites grow vertically, perpendicular to the anode-

7 electrolyte interface. Lithium-indium dendrites grow laterally in stripes, much denser

8 and much more uniform than Li dendrites. This is because the growth of Li dendrites is

9 induced by the non-uniform Li deposition that prefers to form whiskers and Li-In dendrites is caused by the volume expansion and slight interface reaction. Therefore,

11 Li-In dendrites have a slower growth rate during cycling. This inspires us that 12 converting $\mathrm{Li}$ deposition morphology might be a novel strategy to realize the application of Li metal anode. Secondly, they have different wettability with electrolyte.

14 The growth of Li dendrites causes many cracks and voids in the electrolyte due to the stress concentration and high reactivity, which leads to a loose and porous electrolyte 
1

structure. But for metal Li-In anode, the formation of thin interphase layer $(15 \mathrm{~nm})$ due to the slight interfacial reaction enables the great wettability between LiIn-LPSCl interface and the dense structure of electrolyte could be achieved. The schematic diagram of Li-LPSCl and LiIn-LPSCl interface evolution in cells before and after cycling is shown in Figure 7. The suppression of Li-In dendrites and the morphological modification of Li dendrites warrant further investigation.
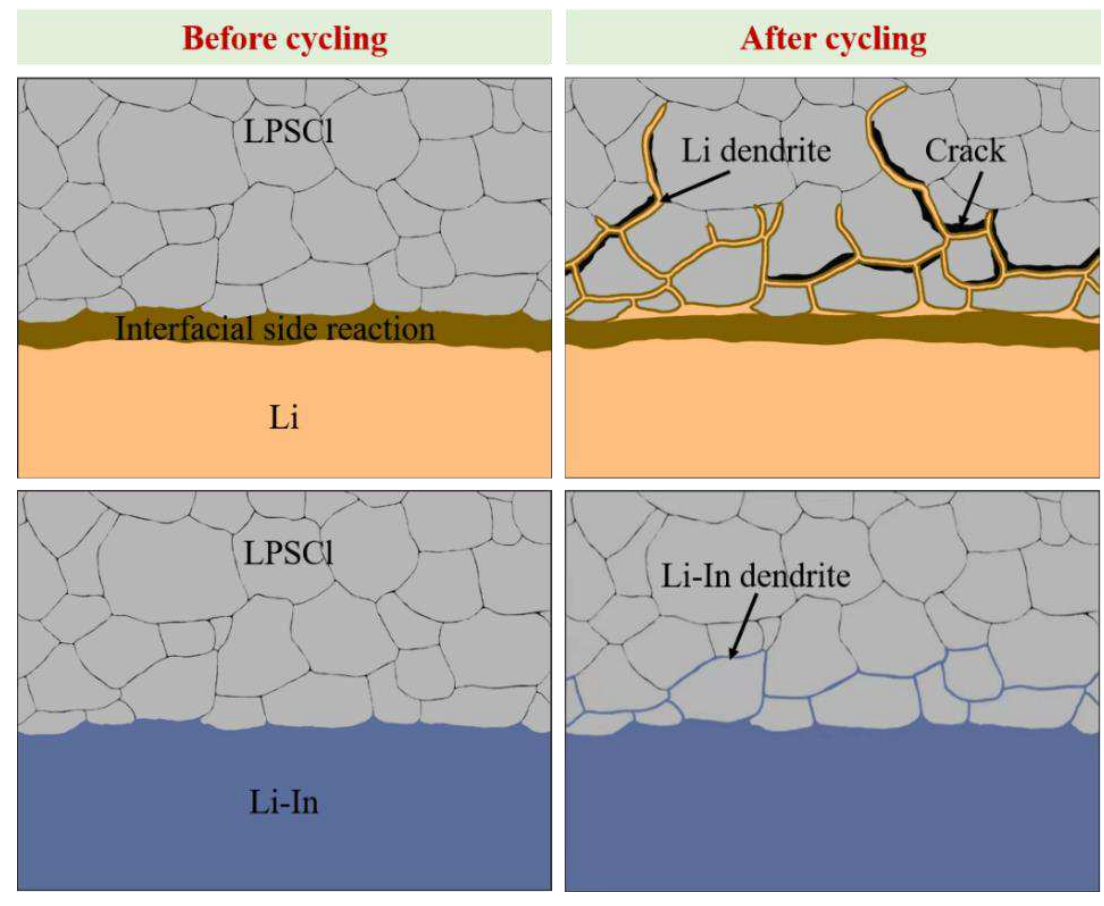

Figure 7. Schematic diagram of Li-LPSCl and LiIn-LPSCl interface evolution in cells before and after cycling.

\section{Conclusion}

In summary, we discovered the growth of Li-In dendrites in sulfide electrolyte and clarified the dendrite morphology and growth mechanism using SEM-EDX analysis, STEM-EELS analysis and AIMD simulations. Our finding suggests that metal In is thermodynamically unstable towards sulfide electrolytes and possibly produces InS at the LiIn-LPSCl interface. When the cell cycled at a high current, the accompanied 
1 volume change and slight interfacial reaction will cause the growth of Li-In dendrites

2 enclosing electrolyte particles, eventually leading to short circuit and cell death after a

3 long cycling. Compared with vertically growing Li dendrites (Figure 6b), laterally

4 striped Li-In dendrites (Figure 3b) are favorable for reducing the growth rate of

5 dendrites and alleviating structural damage on sulfide electrolyte, which provides a new

6 strategy for modifying Li metal anode to improve cycling life. Our investigation reveals

7 the failure mechanism of ASSLBs using Li-In anode and gives a valuable insight on the

8 Li-In dendrites, which will provide rational guidance for testing the performance of

9 sulfide-based ASSLBs.

\section{Methods}

Material synthesis: Sulfide solid electrolyte LPSCl was produced by GLESI, CHINA. Li 2 S ( $>99.9 \%$ purity, Alfa Aesar), $\mathrm{P}_{2} \mathrm{~S}_{5}$ ( $>99 \%$ purity, Alfa Aesar) and $\mathrm{LiCl}$ ( $>99.9 \%$ purity, Alfa Aesar) were first mixed in an appropriate molar ratio. The mixture was then placed in a $\mathrm{ZrO}_{2}$ pot containing a $\mathrm{ZrO}_{2}$ ball to be mechanically milled using the planetary ball milling apparatus at 500 r.p.m. for 30 hours. Following the ball milling procedure, the mixture was heated at $550{ }^{\circ} \mathrm{C}$ for 6 hours under argon (Ar) atmosphere with $\mathrm{H}_{2} \mathrm{O}$ and $\mathrm{O}_{2}<0.1$ ppm.

$\mathrm{LiNbO}_{3}$ coated $\mathrm{LiNi}_{0.6} \mathrm{Co}_{0.2} \mathrm{Mn}_{0.2} \mathrm{O}_{2}$ (LNO@NCM622) was provided by GLESI, CHINA. By means of the fluidized bed technology, the LNO coating layer was formed with an ethanol solution of $\mathrm{Li}$ and $\mathrm{Nb}$. Metal $\mathrm{Li}$ and niobium ethoxide ( $>99.9 \%$ purity, Alfa Aesar; molar ratio, $\mathrm{Li}: \mathrm{Nb}=1: 1$ ) were dissolved in anhydrous ethanol (Kanto Chemical) under Ar atmosphere. The solution was sprayed onto NCM622 particles at a 
1 spraying rate of $2 \mathrm{~g} \mathrm{~min}^{-1}$ by a rolling fluidized coating machine (Powrex, MP-01). The

2 LNO@NCM622 was finally heated at $400{ }^{\circ} \mathrm{C}$ for 60 min under $\mathrm{O}_{2}$. The coating layer

3 thickness can be controlled by spraying duration. Lithium-indium (Li-In) alloy was

4 prepared by solid-state diffusion method. Li metal and In metal with a weight ratio of

$5 \quad$ 2:98 was combined under a $760 \mathrm{Mpa}$ pressure.

Fabrication of all-solid-state lithium battery: As-synthesized LNO@NCM622 powder was mixed with LPSCl electrolyte powder in a mass ratio of 70:30 using an agate mortar, and the obtained composite was used as cathode for the full cell. Li-In alloy foil was used as anode. Cells were assembled as follow: $80 \mathrm{mg}$ LPSCl electrolyte powder was pressed under $150 \mathrm{MPa}$ into an electrolyte layer with a diameter of $10 \mathrm{~mm}$. Then, $40 \mathrm{mg}$ composite cathode powder was uniformly spread on one side of the electrolyte layer and then a stainless steel (SS) foil was put on composite cathode layer. Next, Li-In alloy $(\sim 25 \mathrm{~g})$ was placed on the other side of the electrolyte layer with a SS foil as collector. Finally, all the components were compressed together under 760 $\mathrm{MPa}$ to form full cell. For the reference cell LNO@NCM622//LPSCl//Li, the Li-In anode was replaced by Li metal ( $\sim \mu$ m thickness) and the pressure was changed from $760 \mathrm{MPa}$ to $100 \mathrm{Mpa}$ with other conditions remaining consistent.

Materials Characterizations: X-ray diffraction pattern (XRD, Rigaku D/MAX-500, Japan) of the LPSCl electrolyte was recorded by using $\mathrm{Cu} \mathrm{K} \alpha$ radiation in the $2 \theta$ range of $10-60^{\circ}$ with a step size of $0.02^{\circ}$. Scanning electron microscope (SEM) equipped with energy-dispersive X-ray spectroscopy (EDX) (JEOL, JSM-7900F, Japan) was used to analyze the cross-sectional morphology of the cell. The sample cross section was 
1 polished using a cross section polisher (JEOL, IB-19520CCP, Japan) before SEM-EDX

2 analysis. The microstructure of the tested sample was characterized by cryo-scanning

3 transmission electron microscope (cryo-STEM, HD2700, Hitachi) at -100 ${ }^{\circ} \mathrm{C}$. Element

4 distribution was observed via EDX (XMAXN 100TLE, Oxford) and component

5 analysis was performed using electron energy loss spectroscopy (Enfinlum, Gatan).

6 STEM specimens were obtained by thinning the electrolyte layer with dual-beam

7 focused ion beam equipment (FIB, NB5000, Hitachi) operated at 2-30 kV. A special

8 compatible holder was used to enable the sample directly transferred from the FIB

9 equipment to cryo-STEM without exposure in the air.

10 Electrochemical measurements: The conductivity of LPSCl was measured on a

$11 \mathrm{SS} / \mathrm{LPSCl} / \mathrm{SS}$ cell using electrochemical workstation (EIS, Bio-Logic VSP-300) over a

12 frequency range of $6 \mathrm{MHz}-1 \mathrm{~Hz}$ at room temperature. For the long-term cycling, the 13 cell LNO@NCM622//LPSCl//Li-In was charged to 3.68V (vs. Li-In/Li ${ }^{+}, 4.3 \mathrm{~V}$ vs. $\left.14 \mathrm{Li} / \mathrm{Li}^{+}\right)$at a constant current mode (1C) and then charged at constant voltage mode (3.68 $15 \mathrm{~V}$ ) for 15 minutes. After that, the cell was discharged at $1 \mathrm{C}$ to $2.1 \mathrm{~V}$ (vs. $\mathrm{Li}-\mathrm{In} / \mathrm{Li}^{+}, 2.72$ respectively.

AIMD calculations: AIMD simulations are performed in the Vienna Ab initio Simulation Package (VASP) with the projector augmented wave (PAW) method. The exchange-correlation potential is described by the generalized gradient approximation (GGA) function, which is parameterized with Perdew-Burke-Ernzerhof(PBE). $500 \mathrm{eV}$ of cutoff energy for plane-wave is set, and $1 \times 1 \times 1 \mathrm{~K}$-point mesh in the first Brillouin 
zone is used as the large atom numbers $(>250)$ of the interfacial models. NVT ensemble based on a Nosé thermostat and time step of 2 fs are adopted in the AIMD simulations.

\section{Data availability}

The data that support the findings of this study are available from the corresponding author upon reasonable request.

\section{References}

1. Goodenough J. B., Park K. S. The Li-ion rechargeable battery: A perspective. J. Am. Chem. Soc. 135, 1167-1176 (2013).

2. Takada K. Progress and prospective of solid-state lithium batteries. Acta. Mater. 61, 759-770 (2013).

3. Lau J., DeBlock R. H., Butts D. M., Ashby D. S., Choi C. S., Dunn B. S. Sulfide solid electrolytes for lithium battery applications. Adv. Energy. Mater. 8, (2018).

4. Tan D. H. S., Banerjee A., Chen Z., Meng Y. S. From nanoscale interface characterization to sustainable energy storage using all-solid-state batteries. Nat. Nanotechnol. 15, 170-180 (2020).

5. Lee Y. G., et al. High-energy long-cycling all-solid-state lithium metal batteries enabled by silvercarbon composite anodes. Nat. Energy 5, 348-348 (2020).

6. Wenzel S., Leichtweiss T., Kruger D., Sann J., Janek J. Interphase formation on lithium solid electrolytes-An in situ approach to study interfacial reactions by photoelectron spectroscopy. Solid State Ionics 278, 98-105 (2015).

7. Wenzel S., Weber D. A., Leichtweiss T., Busche M. R., Sann J., Janek J. Interphase formation and degradation of charge transfer kinetics between a lithium metal anode and highly crystalline $\mathrm{Li}_{7} \mathrm{P}_{3} \mathrm{~S}_{11}$ solid electrolyte. Solid State Ionics 286, 24-33 (2016).

8. Wenzel S., Sedlmaier S. J., Dietrich C., Zeier W. G., Janek J. Interfacial reactivity and interphase growth of argyrodite solid electrolytes at lithium metal electrodes. Solid State Ionics 318, 102-112 (2018).

9. Porz L., et al. Mechanism of lithium metal penetration through inorganic solid electrolytes. Adv. Energy Mater. 7, (2017).

10. Kasemchainan J., et al. Critical stripping current leads to dendrite formation on plating in lithium anode solid electrolyte cells. Nat. Mater. 18, 1105-1112 (2019).

11. Schlenker R., et al. Understanding the lifetime of battery cells based on solid-state $\mathrm{Li}_{6} \mathrm{PS}_{5} \mathrm{Cl}$ electrolyte paired with lithium metal electrode. ACS Appl. Mater. Inter. 12, 20012-20025 (2020).

12. Obrovac M. N., Chevrier V. L. Alloy negative electrodes for Li-ion batteries. Chem. Rev. 114, 1144411502 (2014).

13. Kanno R., et al. A self-assembled breathing interface for all-solid-state ceramic lithium batteries. 
Electrochem. Solid St. 7, A455-A458 (2004).

14. Nagao M., Hayashi A., Tatsumisago M. Bulk-type lithium metal secondary battery with indium thin layer at interface between $\mathrm{Li}$ electrode and $\mathrm{Li}_{2} \mathrm{~S}-\mathrm{P}_{2} \mathrm{~S}_{5}$ solid electrolyte. Electrochemistry 80, 734-736 (2012).

15. Santhosha A. L., Medenbach L., Buchheim J. R., Adelhelm P. The indium-lithium electrode in solidstate lithium-ion batteries: phase Formation, redox potentials, and interface stability. Batteries Supercaps 2, 524-529 (2019).

16. Sang L. Z., et al. Understanding the effect of interlayers at the thiophosphate solid electrolyte/lithium interface for all-solid-state Li batteries. Chem. Mater. 30, 8747-8756 (2018).

17. Kato A., Kowada H., Deguchi M., Hotehama C., Hayashi A., Tatsumisago M. XPS and SEM analysis between $\mathrm{Li} / \mathrm{Li}_{3} \mathrm{PS}_{4}$ interface with Au thin film for all-solid-state lithium batteries. Solid State Ionics $\mathbf{3 2 2}$, $1-4$ (2018).

18. Kato A., Hayashi A., Tatsumisago M. Enhancing utilization of lithium metal electrodes in all-solidstate batteries by interface modification with gold thin films. J. Power Sources 309, 27-32 (2016).

19. Kato A., et al. High-temperature performance of all-solid-state lithium-metal batteries having Li/Li $\mathrm{PS}_{4}$ interfaces modified with Au thin films. J. Electrochem. Soc. 165, A1950-A1954 (2018).

20. Sakuma M., Suzuki K., Hirayama M., Kanno R. Reactions at the electrode/electrolyte interface of all-solid-state lithium batteries incorporating $\mathrm{Li}-\mathrm{M}(\mathrm{M}=\mathrm{Sn}, \mathrm{Si})$ alloy electrodes and sulfide-based solid electrolytes. Solid State Ionics 285, 101-105 (2016).

21. Huggins R. A. Lithium alloy negative electrodes. J. Power Sources 81, $13-19$ (1999).

22. Wang J. Q., Raistrick I. D., Huggins R. A. Behavior of some binary lithium alloys as negative electrodes in organic solvent-based electrolytes. J. Electrochem. Soc. 133, 457-460 (1986).

23. Santosh K. C., Xiong K., Longo R. C., Cho K. Interface phenomena between Li anode and lithium phosphate electrolyte for Li-ion battery. J. Power Sources 244, 136-142 (2013).

24. Zhu Y.Z., He X. F., Mo Y. F. Origin of outstanding stability in the lithium solid electrolyte materials: Insights from thermodynamic analyses based on first-principles calculations. ACS Appl. Mater. Inter. 7, 23685-23693 (2015).

25. Cao D. X., et al. Stable thiophosphate-based all-solid-state lithium batteries through conformally interfacial nanocoating. Nano Lett. 20, 1483-1490 (2020).

26. Whiteley J. M., Taynton P., Zhang W., Lee S. H. Ultra-thin solid-state Li-ion electrolyte membrane facilitated by a self-healing polymer matrix. Adv. Mater. 27, 6922-6927 (2015).

27. Auvergniot J., Cassel A., Ledeuil J. B., Viallet V., Seznec V., Dedryvere R. Interface stability of argyrodite $\mathrm{Li}_{6} \mathrm{PS}_{5} \mathrm{Cl}$ toward $\mathrm{LiCoO}_{2}, \mathrm{LiNi}_{1 / 3} \mathrm{Co}_{1 / 3} \mathrm{Mn}_{1 / 3} \mathrm{O}_{2}$, and $\mathrm{LiMn}_{2} \mathrm{O}_{4}$ in bulk all-solid-state batteries. Chem. Mater. 29, 3883-3890 (2017). 


\section{Figures}
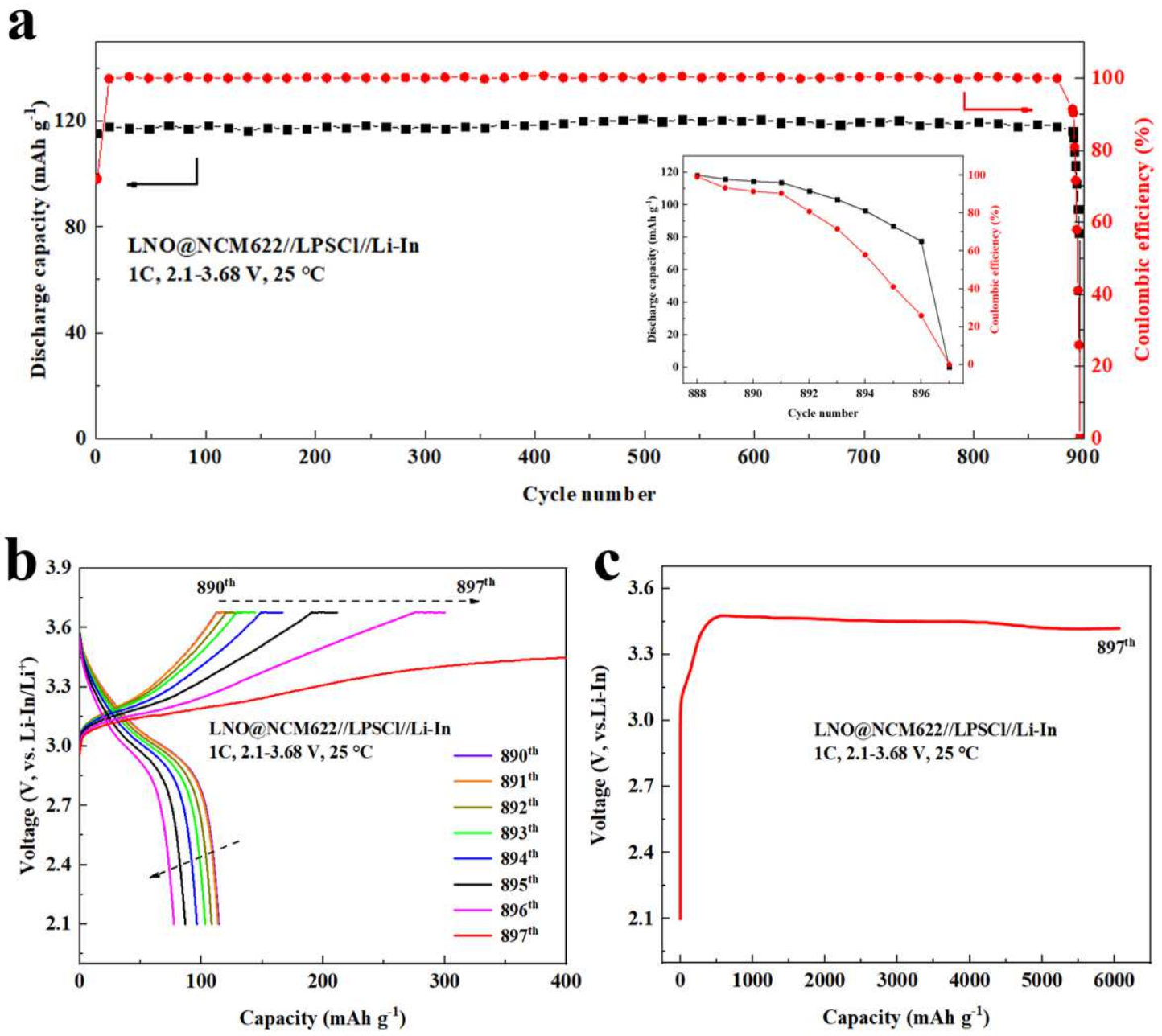

Figure 1. (a) Long-term cycling of the assembled cell LNO@NCM622//LPSCl//Li-In at $1 \mathrm{C}$ at RT. (b) Galvanostatic charge-discharge profiles from the $890^{\text {th }}$ to the $897^{\text {th }}$ cycle.

(c) Galvanostatic charge profile for the $897^{\text {th }}$ cycle. 


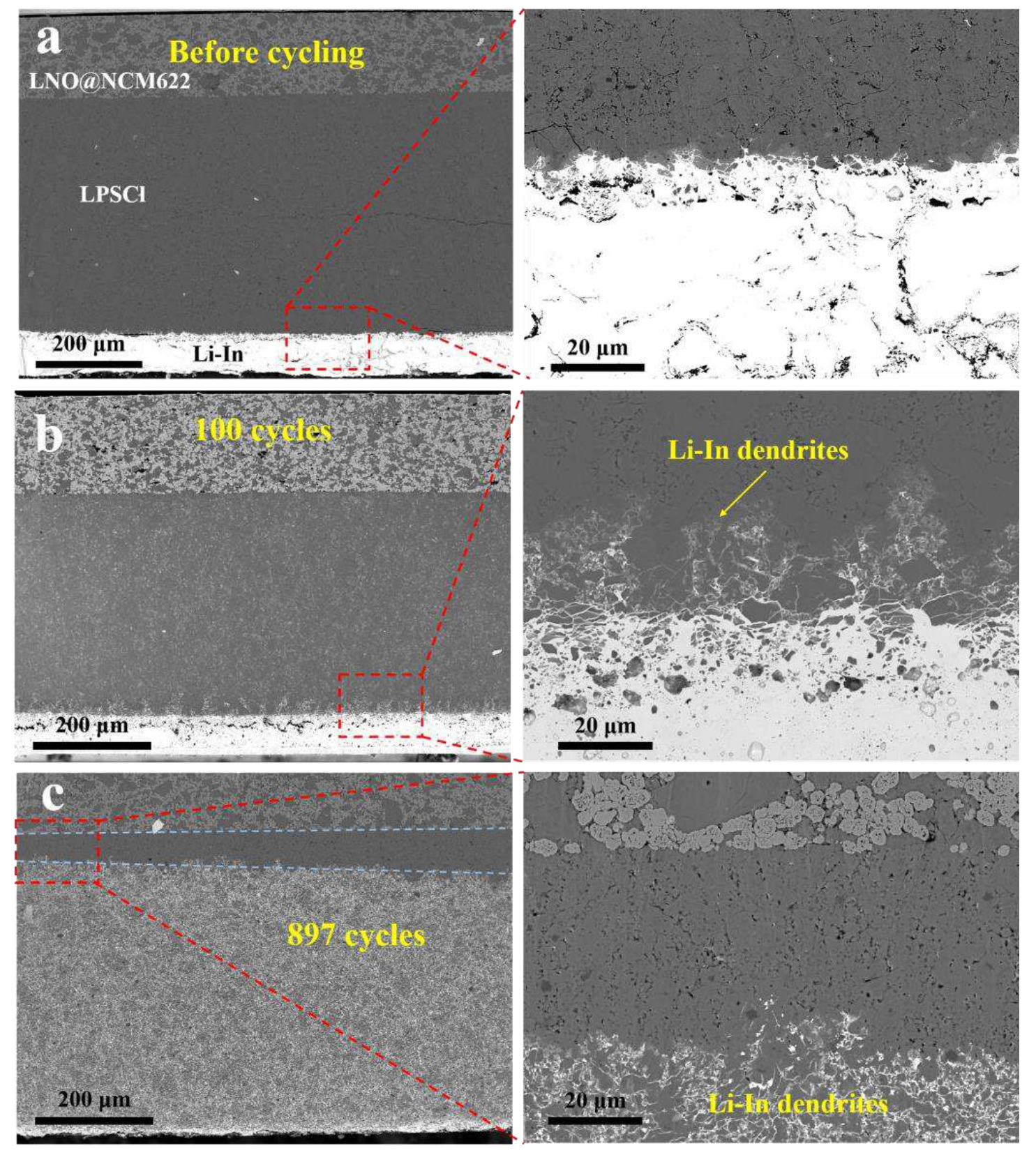

Figure 2. Cross-sectional SEM images for the cell LNO@NCM622//LPSCl//Li-In (a)

before cycling, (b) after 100 cycles and (c) after 897 cycles. 

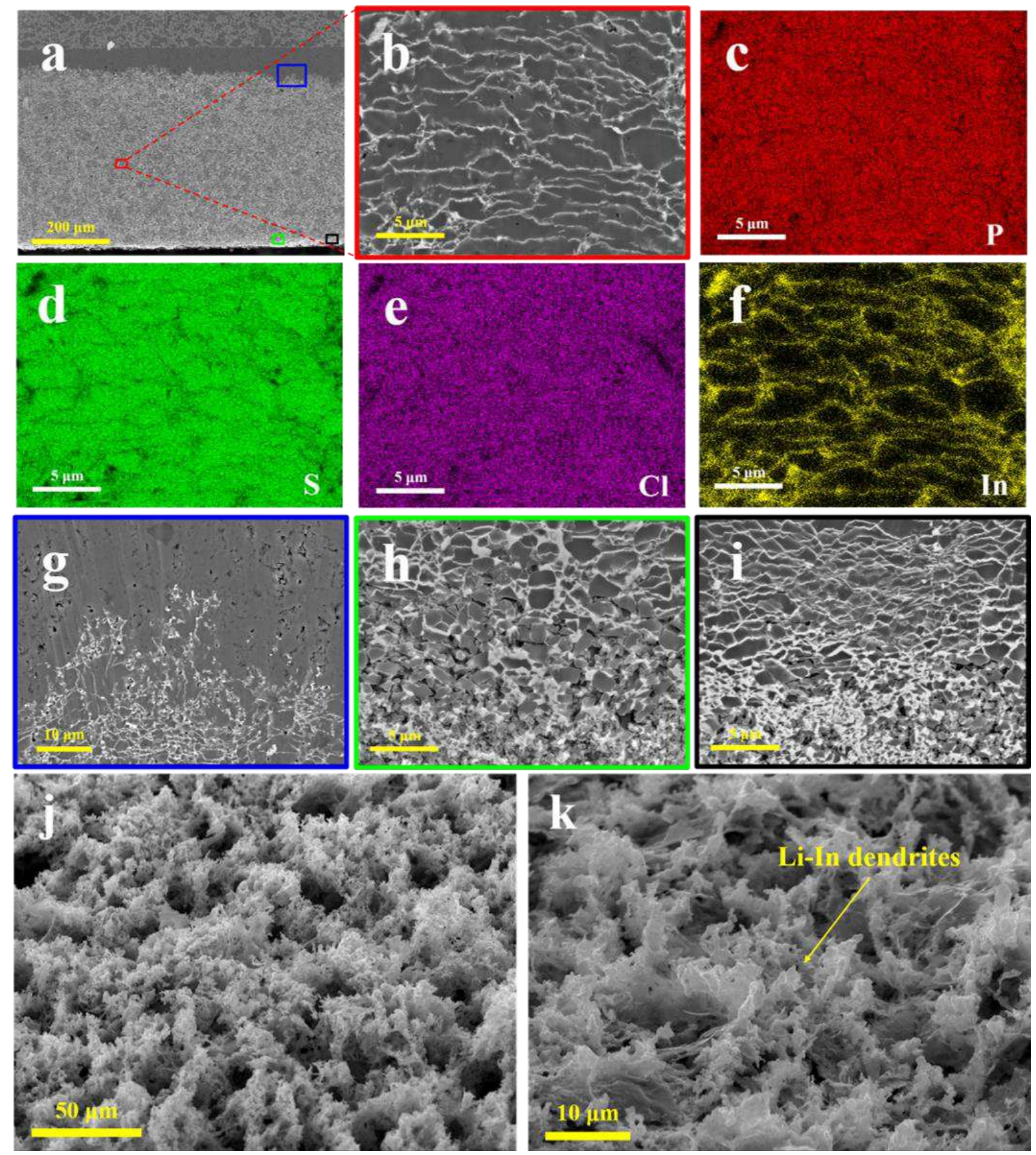

Figure 3. (a) Cross-sectional SEM images of the cell LNO@NCM622//LPSCl//Li-In

after 897 cycles. (b) SEM image and EDX mapping of (c) P, (d) S, (e) $\mathrm{Cl}$ and (f) In at the middle of Li-In dendrites. SEM images at (g) the top of Li-In dendrites, (h) the Inrich layer and (i) the interface between electrolyte and In-rich layer. SEM images of LiIn dendrites from an oblique view after the cell was soaked and washed with the deionized water at (j) low magnification and (k) high magnification. 

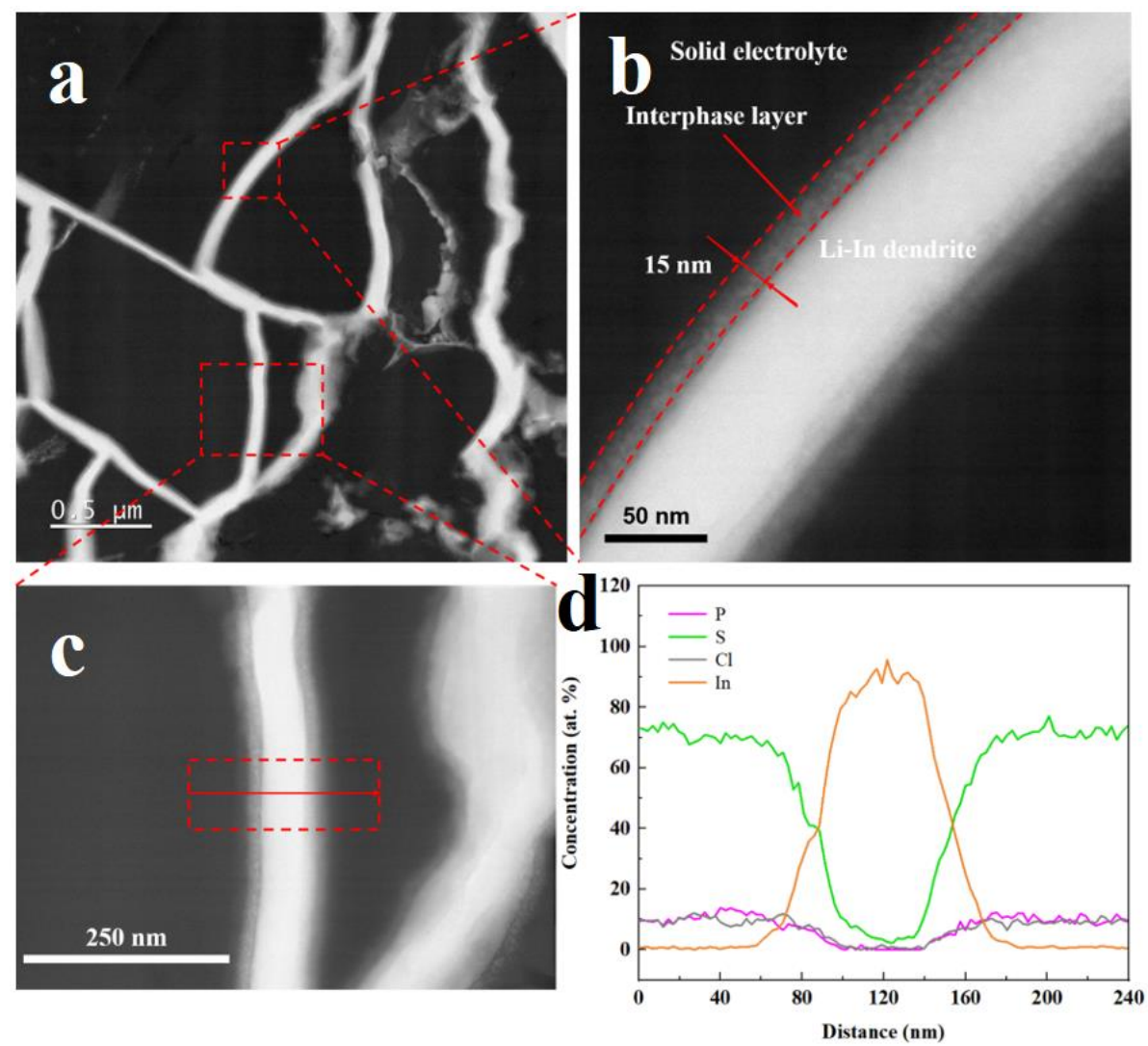

Figure 4. STEM images for (a) Li-In dendrites, (b) magnified Li-In dendrites with interphase layer and (c) magnified Li-In dendrites used for performing EDX. (d) EDX mapping of $\mathrm{P}, \mathrm{S}, \mathrm{Cl}$ and $\mathrm{In}$ in the direction of the red arrow. 

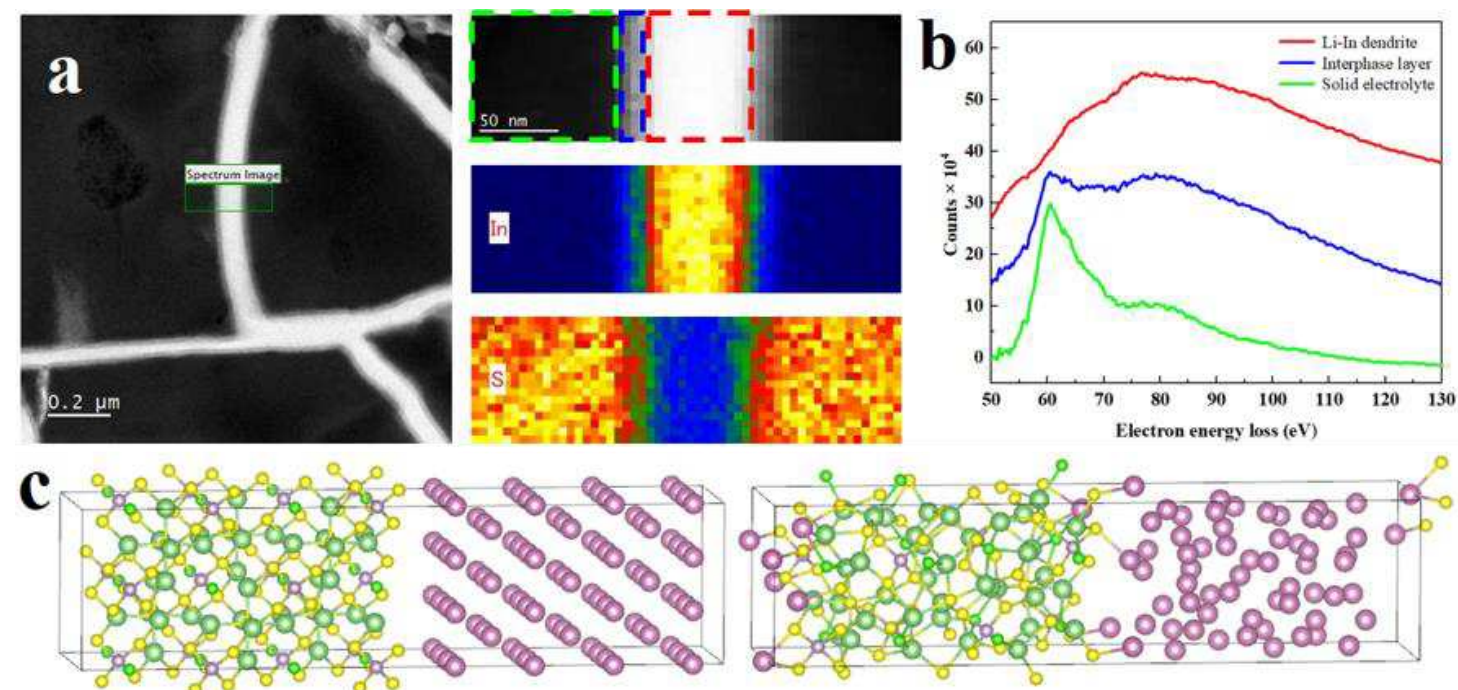

Before MD (0 ps)
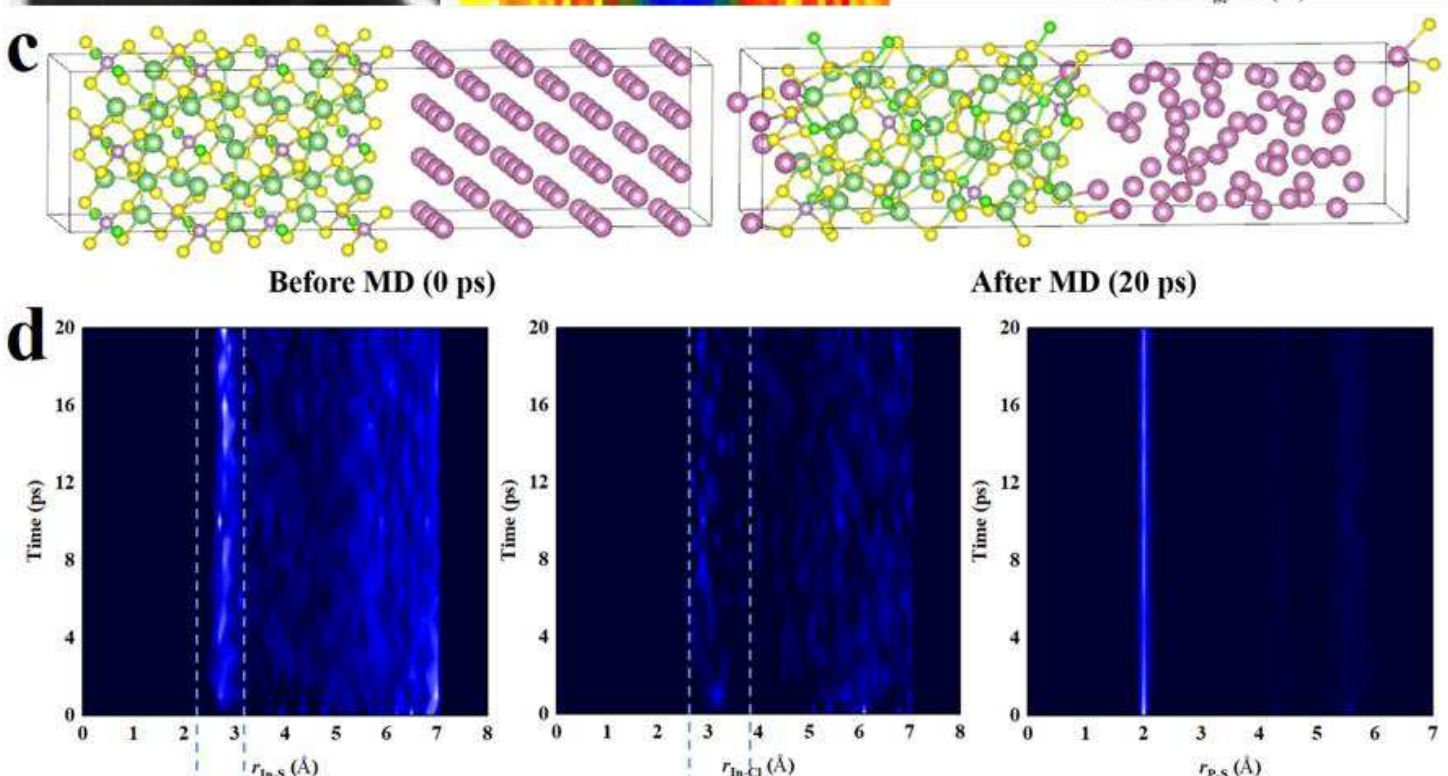

After MD (20 ps)
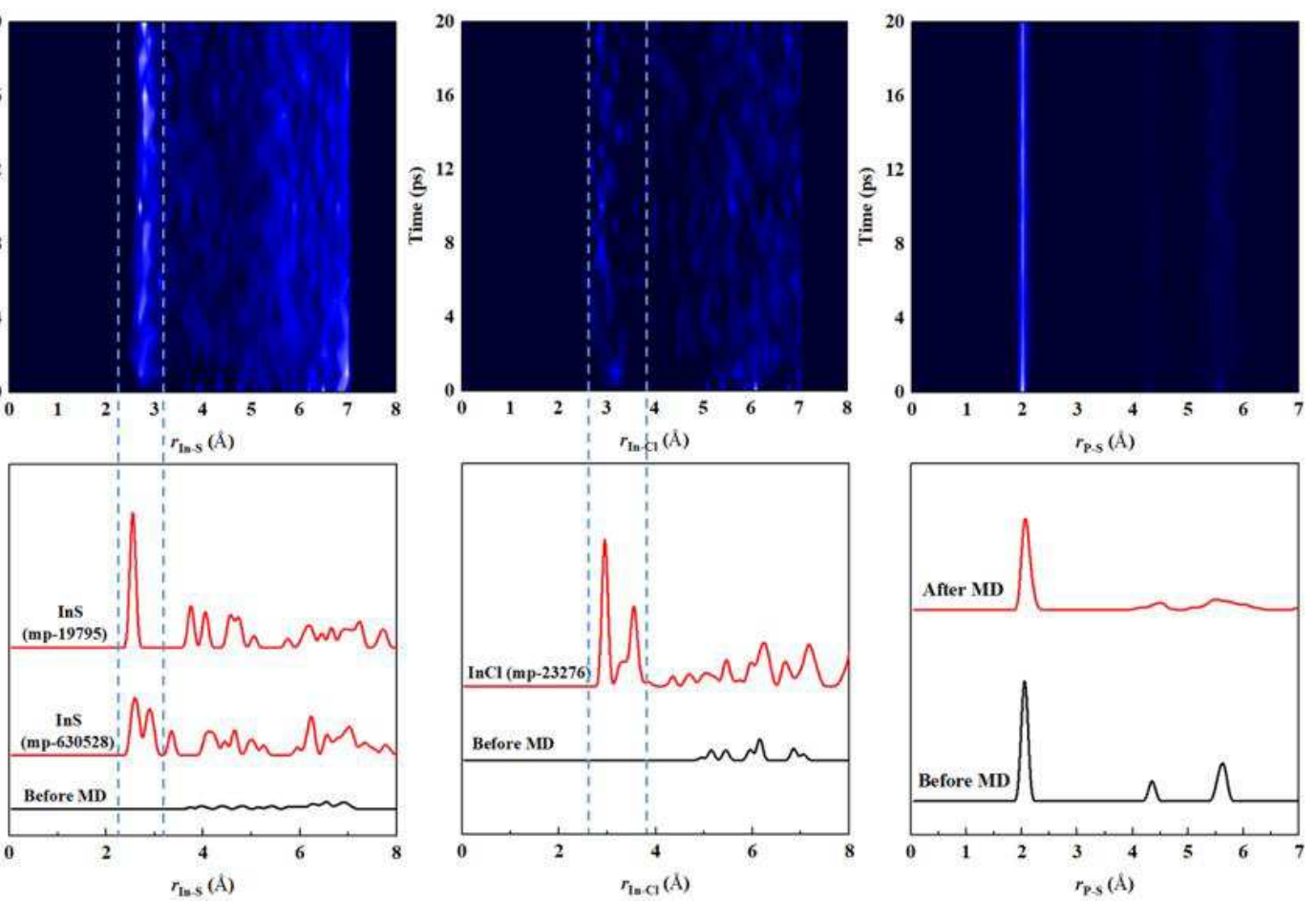

Figure 5. (a) STEM images of Li-In dendrites and corresponding EELS mapping of In and $\mathrm{S}$ for the magnified boxed area. (b) Electron energy loss spectra for Li-In dendrite, LPSCl electrolyte and their interphase layer. (c) LPSCl-In interface models before MD (0 ps) and after MD (20 ps). (d) Evolutions of RDFs of In-S, In-Cl and P-S for LPSClIn interface during simulation and RDFs of In-S, In-Cl and P-S for reference materials ( $\mathrm{InS}, \mathrm{InCl}$ ) with the initial structure (before $\mathrm{MD}$ ) provided as a reference. 

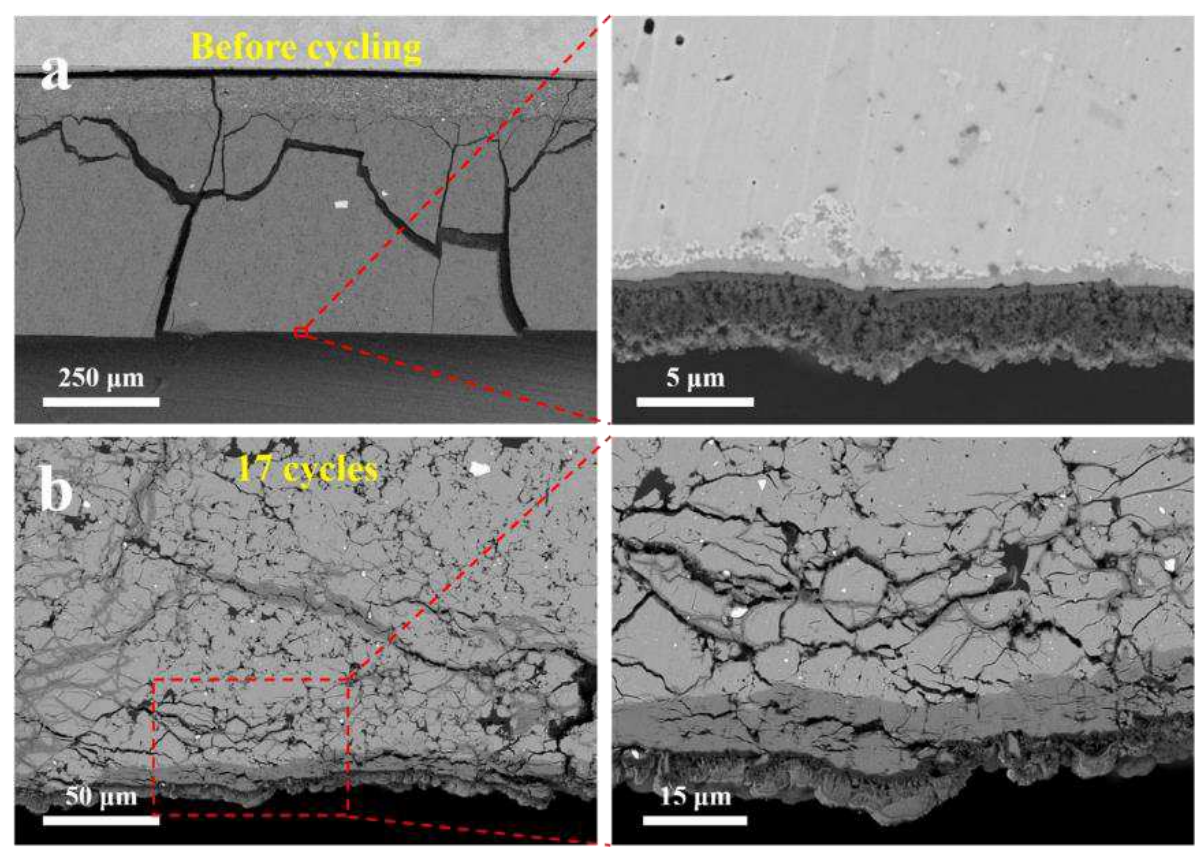

Figure 6. Cross-sectional SEM images of the cell LNO@NCM622//LPSCl//Li (a) before cycling and (b) after 17 cycles. 

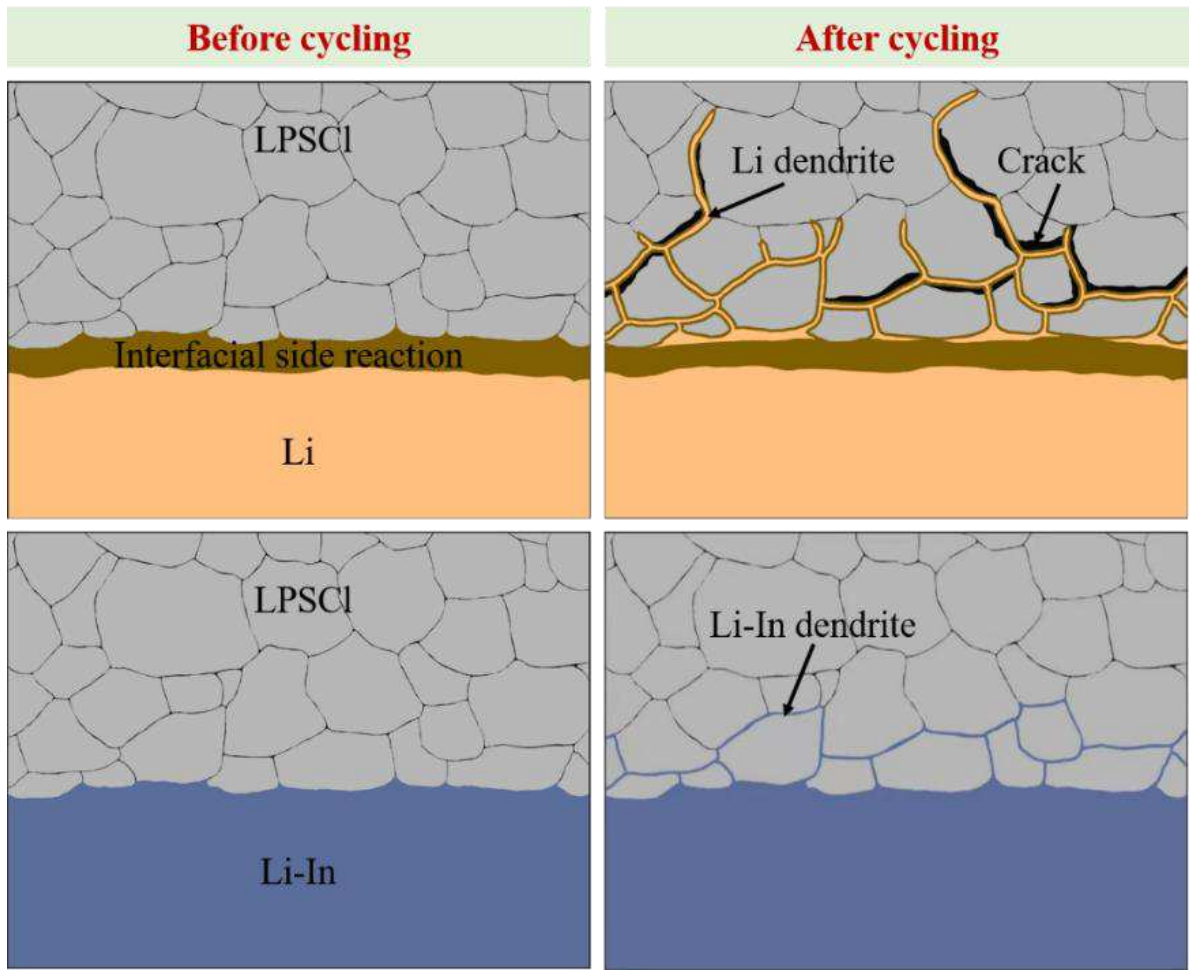

Figure 7. Schematic diagram of Li-LPSCl and LiIn-LPSCl interface evolution in cells before and after cycling. 
Figures

a
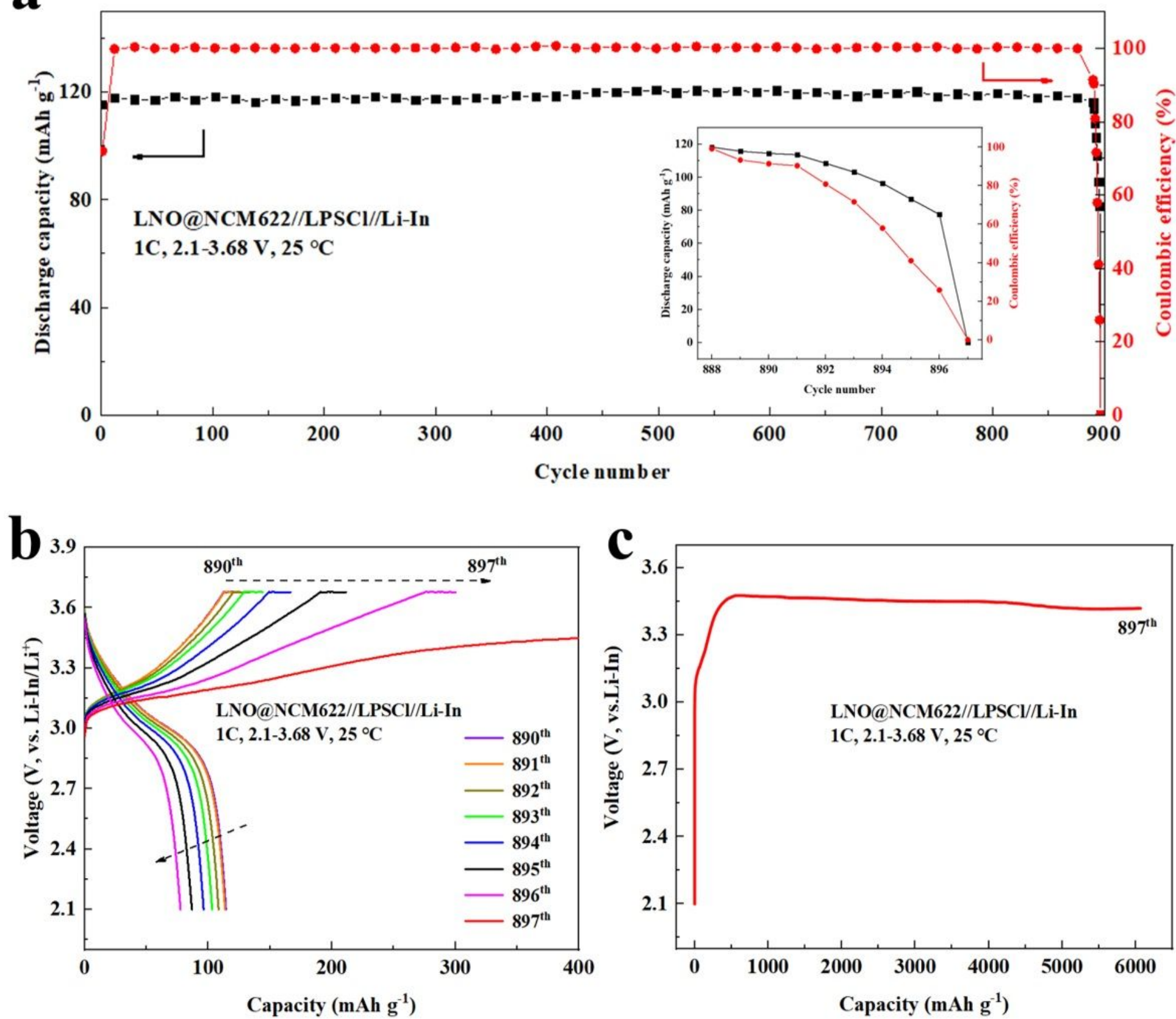

Figure 1

(a) Long-term cycling of the assembled cell LNO@NCM622//LPSCl//Li-In at 1C at RT. (b) Galvanostatic charge-discharge profiles from the 890th to the 897th cycle. (c) Galvanostatic charge profile for the 897th cycle. 


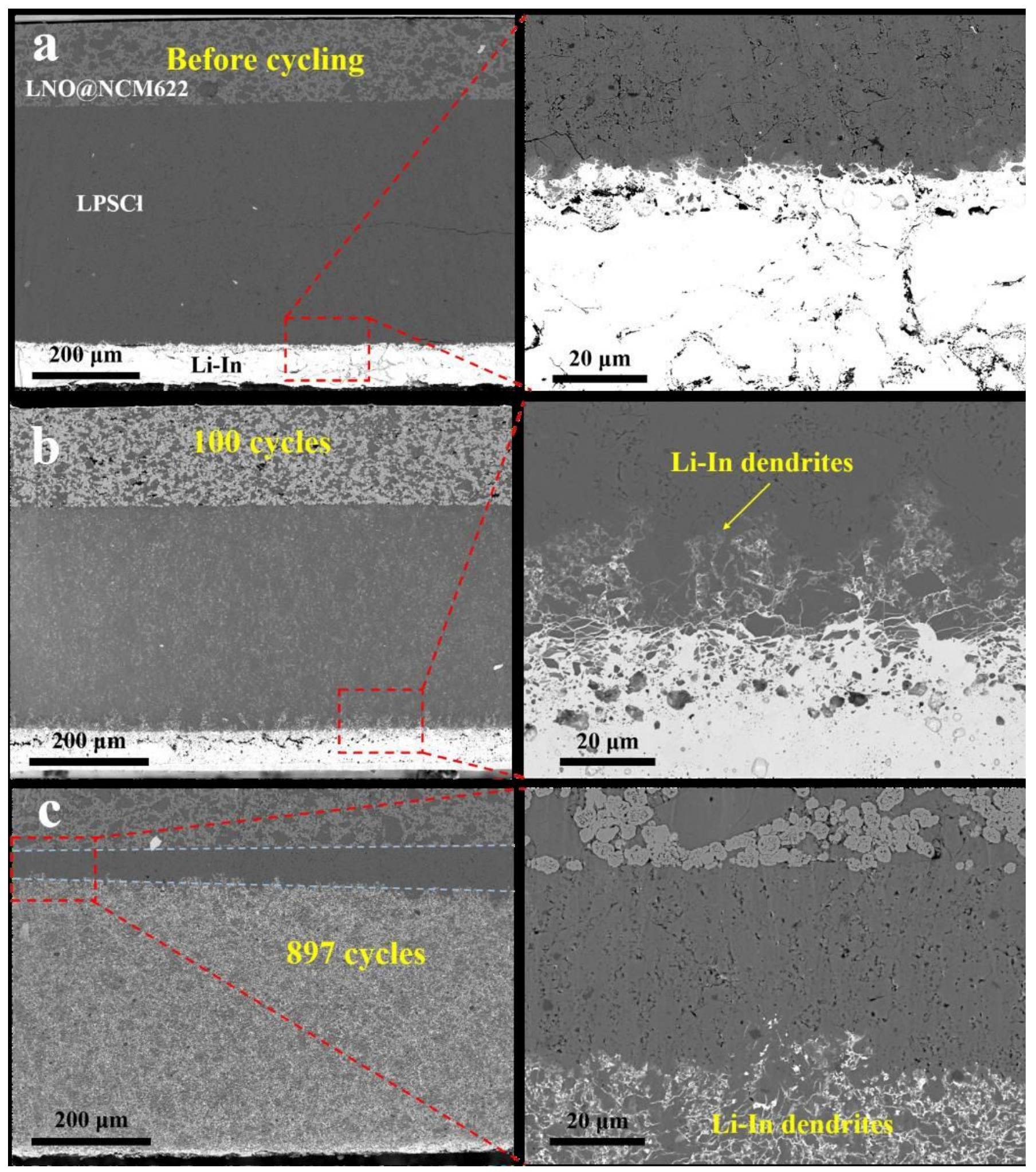

Figure 2

Cross-sectional SEM images for the cell LNO@NCM622//LPSCI//Li-In (a) before cycling, (b) after 100 cycles and (c) after 897 cycles. 


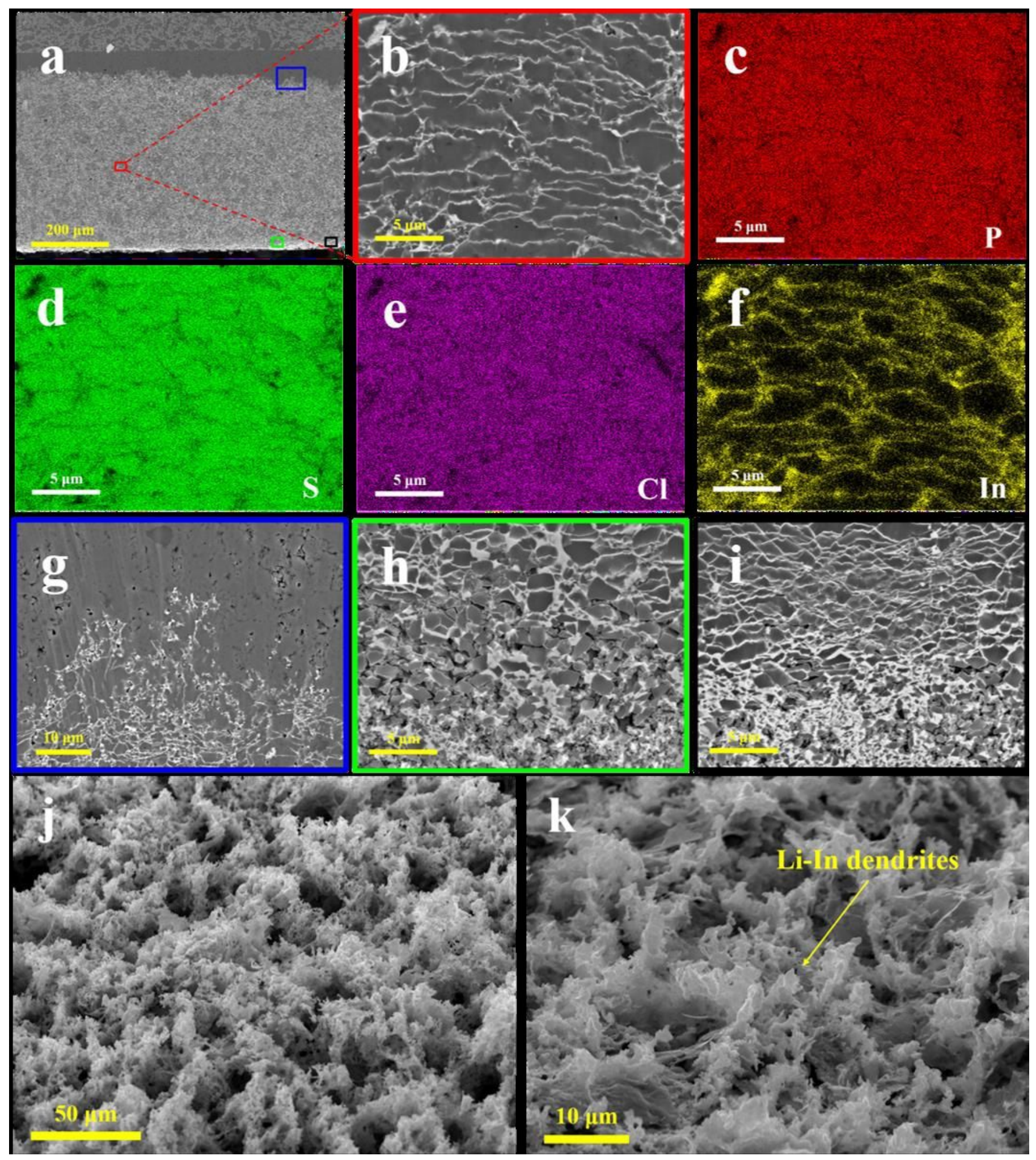

Figure 3

(a) Cross-sectional SEM images of the cell LNO@NCM622//LPSCI//Li-In after 897 cycles. (b) SEM image and EDX mapping of (c) P, (d) S, (e) Cl and (f) In at the middle of Li-In dendrites. SEM images at (g) the top of Li-In dendrites, (h) the In-rich layer and (i) the interface between electrolyte and In-rich layer. SEM images of Li-In dendrites from an oblique view after the cell was soaked and washed with the deionized water at (j) low magnification and ( $k$ ) high magnification. 


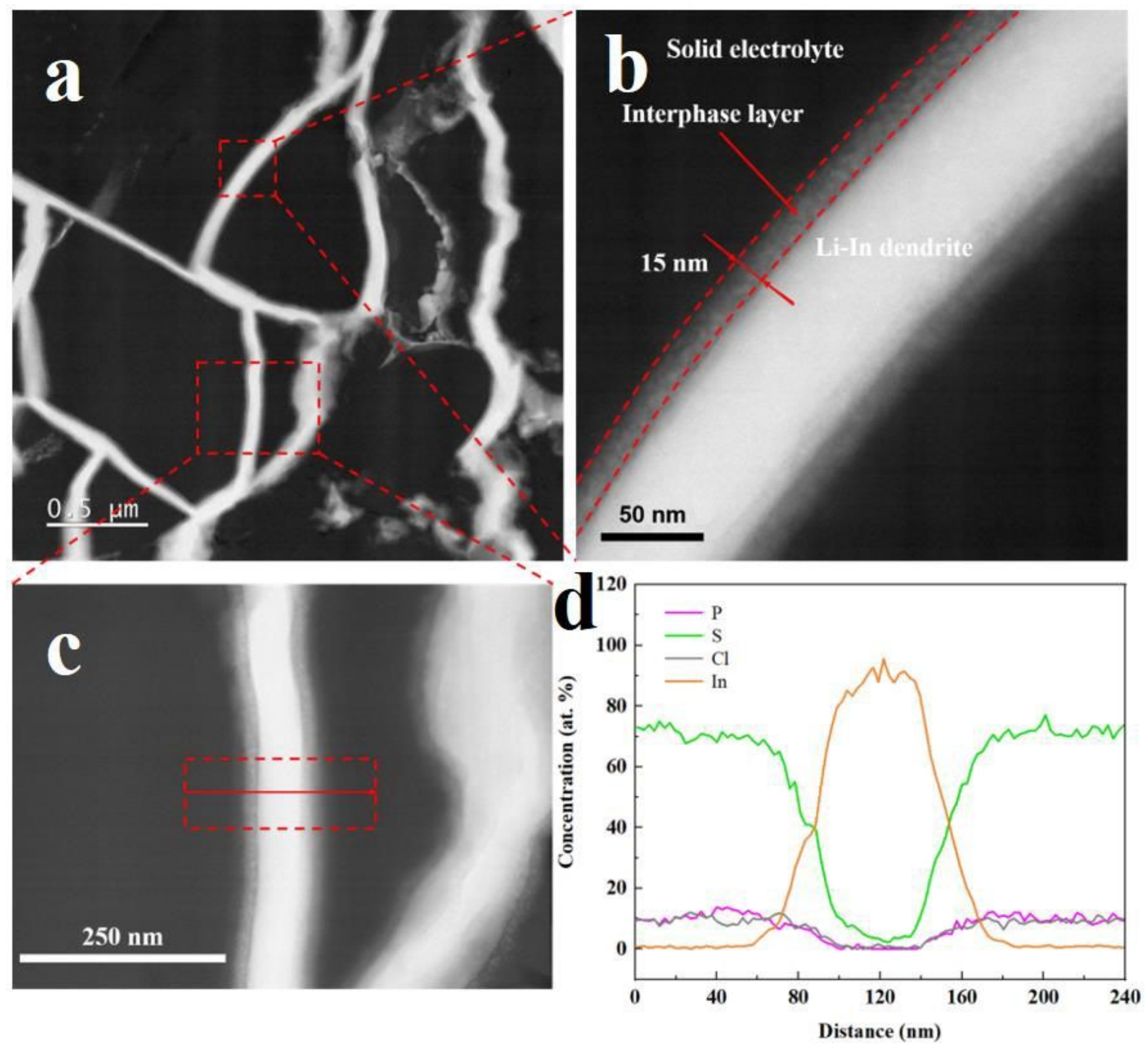

Figure 4

STEM images for (a) Li-In dendrites, (b) magnified Li-In dendrites with interphase layer and (c) magnified $\mathrm{Li}$-In dendrites used for performing EDX. (d) EDX mapping of $\mathrm{P}, \mathrm{S}, \mathrm{Cl}$ and In in the direction of the red arrow. 


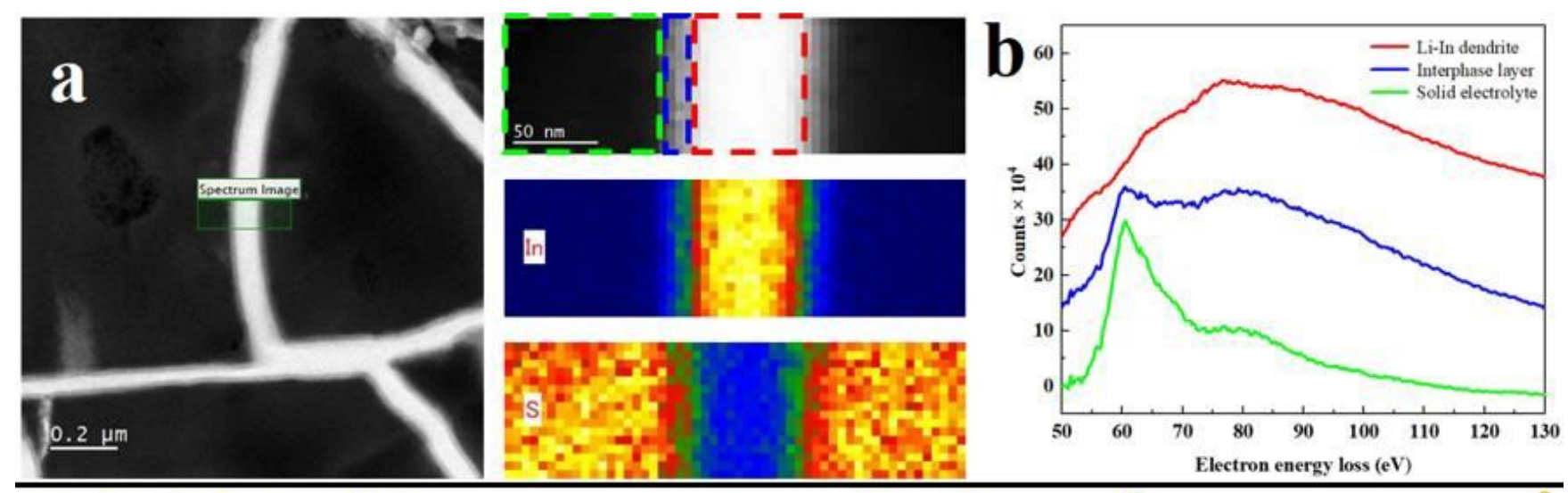

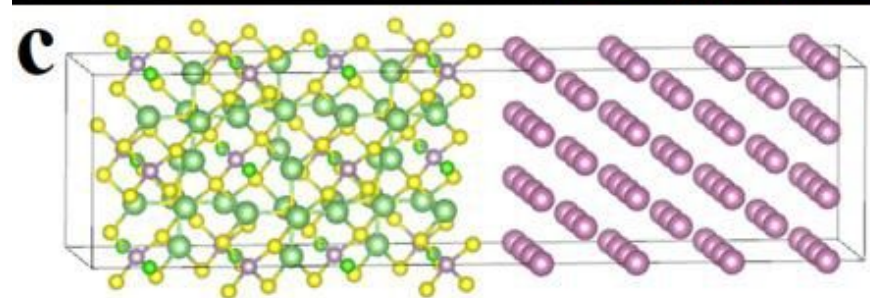

Before MD (0 ps)

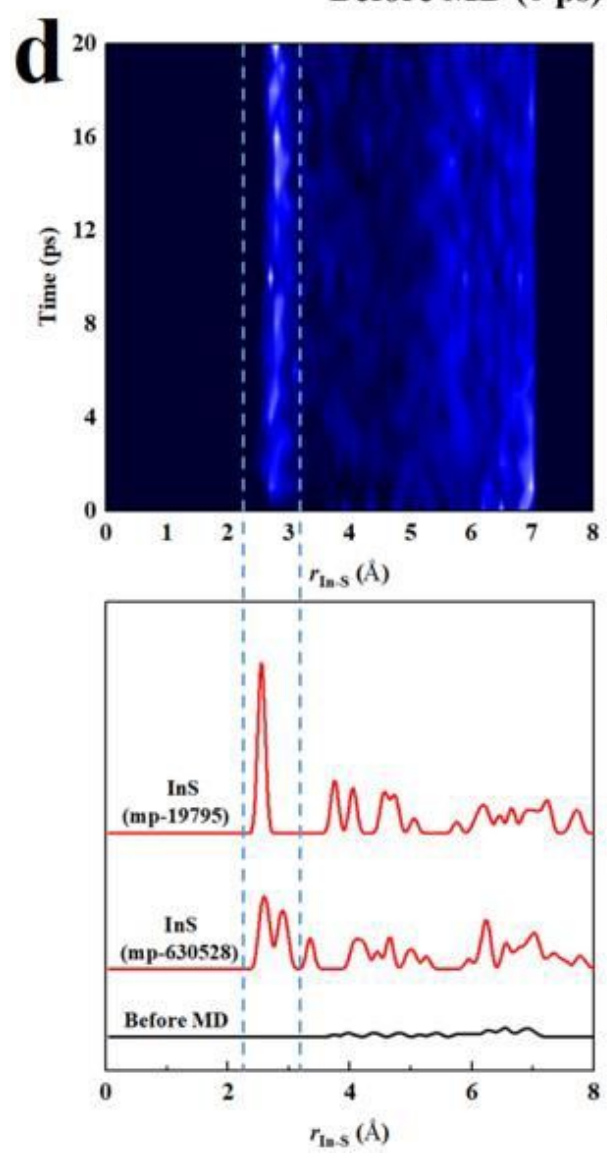

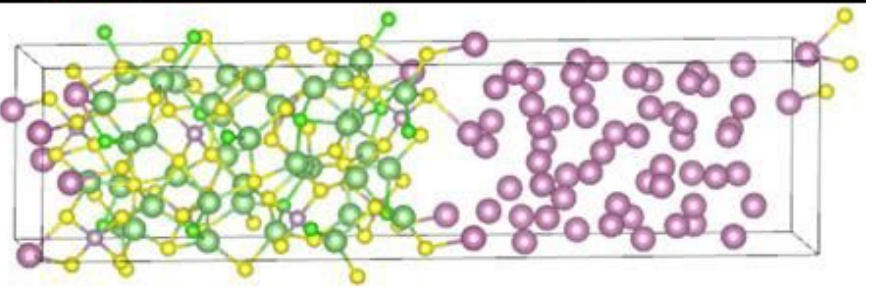

After MD (20 ps)
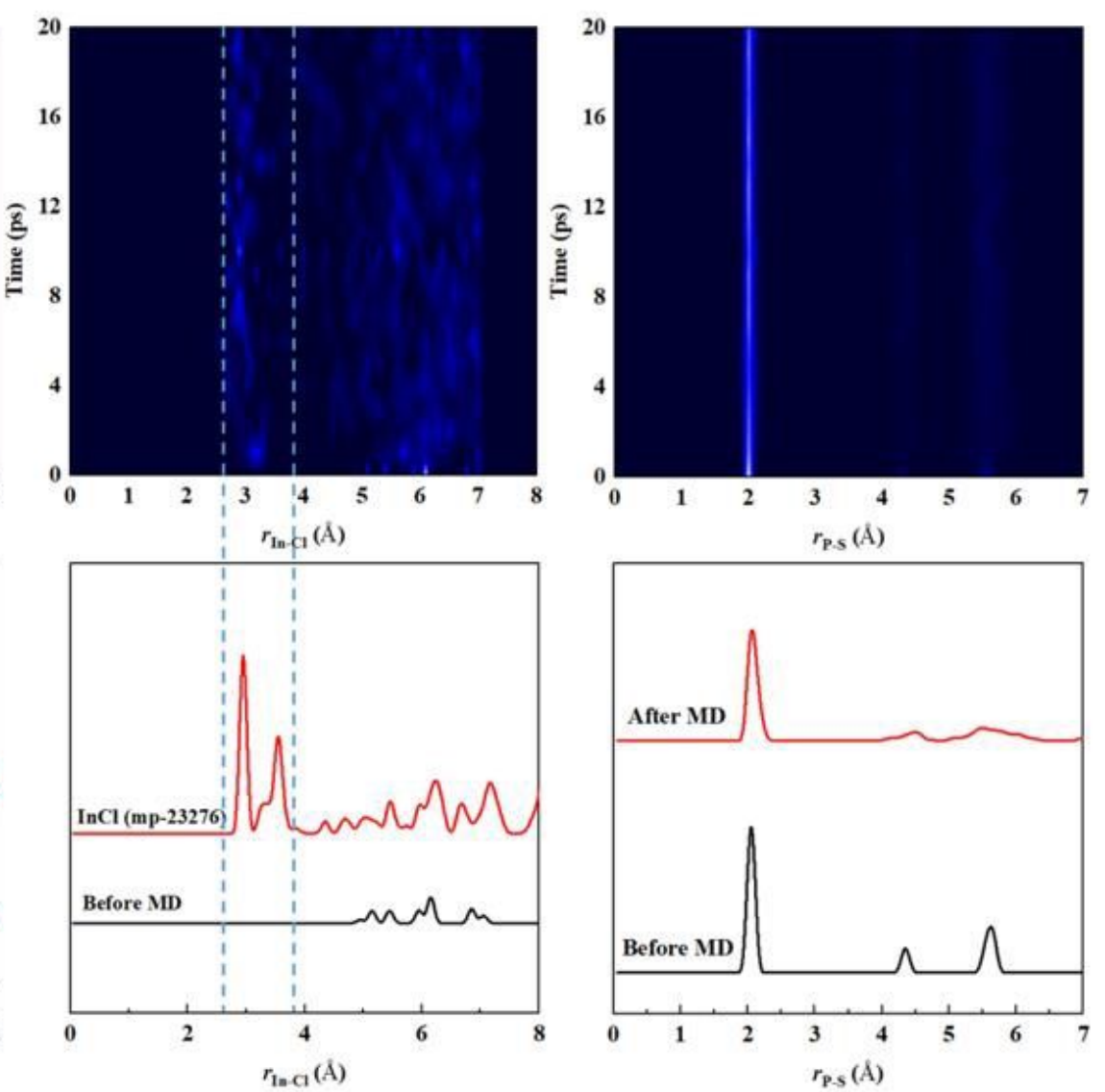

Figure 5

(a) STEM images of Li-In dendrites and corresponding EELS mapping of In and S for the magnified boxed area. (b) Electron energy loss spectra for Li-In dendrite, LPSCI electrolyte and their interphase layer. (c) LPSCl-In interface models before MD (0 ps) and after MD (20 ps). (d) Evolutions of RDFs of In-S, In-Cl and P-S for LPSCl-In interface during simulation and RDFs of In-S, In-Cl and P-S for reference materials (InS, $\mathrm{InCl}$ ) with the initial structure (before MD) provided as a reference. 


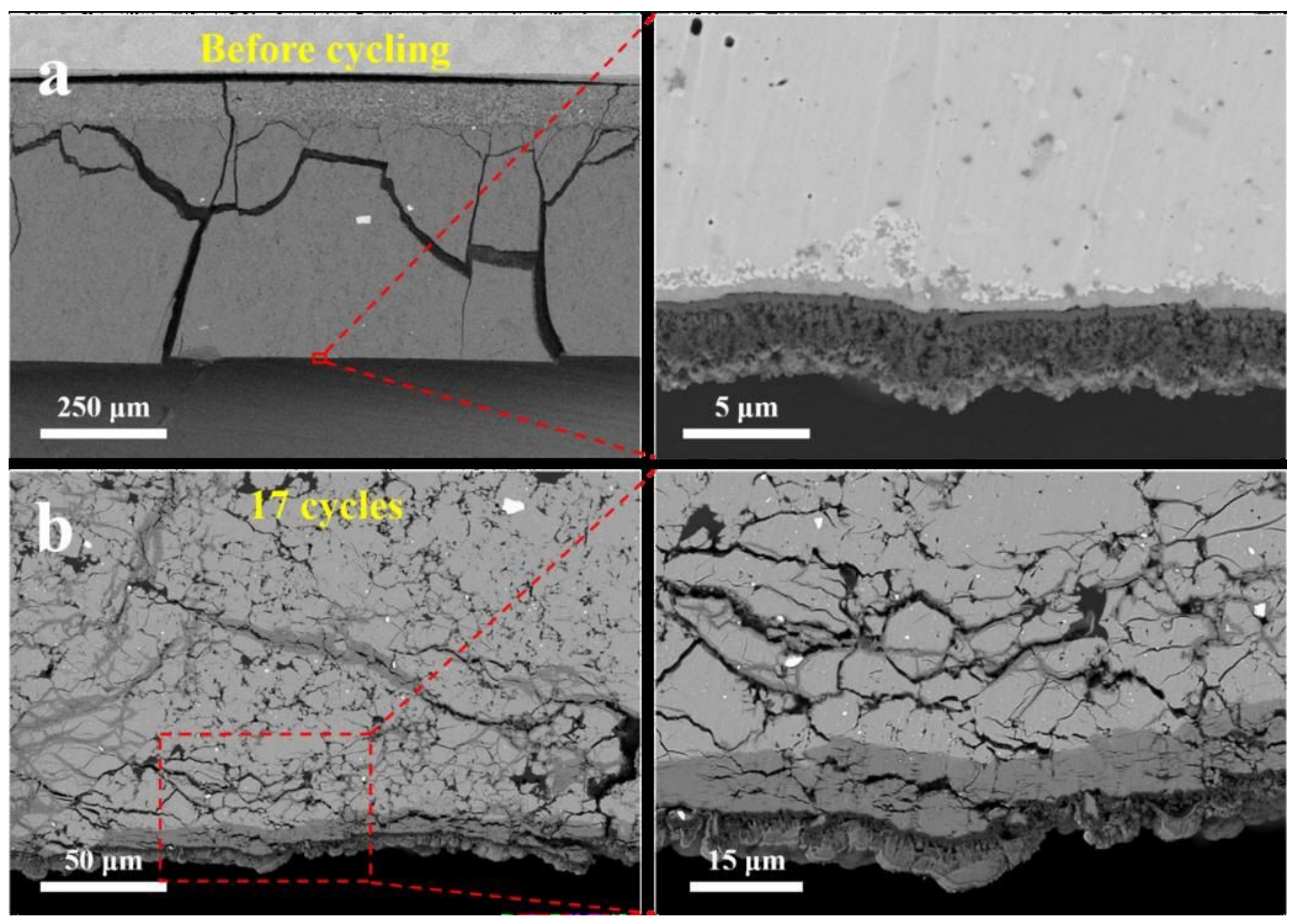

Figure 6

Cross-sectional SEM images of the cell LNO@NCM622//LPSCI//Li (a) before cycling and (b) after 17 cycles. 


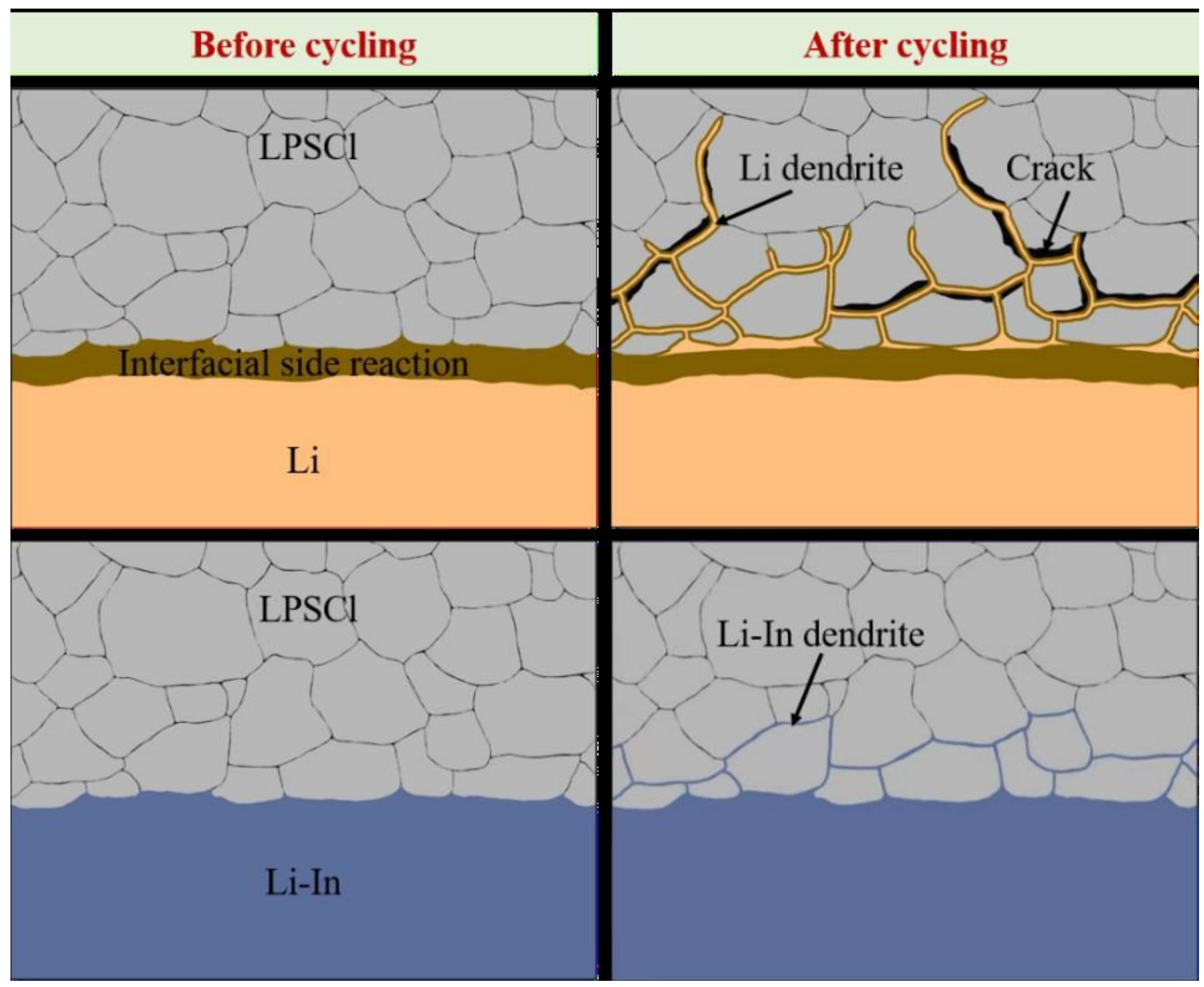

Figure 7

Schematic diagram of Li-LPSCl and Liln-LPSCl interface evolution in cells before and 8 after cycling.

\section{Supplementary Files}

This is a list of supplementary files associated with this preprint. Click to download.

- Supplementaryinformation.pdf 